\title{
1 Experimental colitis drives enteric alpha-synuclein accumulation and Parkinson-like brain
} 2 pathology

4 Stefan Grathwohl ${ }^{1}$, Emmanuel Quansah ${ }^{2}$, Nazia Maroof ${ }^{1}$, Jennifer A. Steiner ${ }^{2}$, Liz Spycher ${ }^{1}$, Fethallah

5 Benmansour ${ }^{3}$, Gonzalo Duran-Pacheco ${ }^{4}$, Juliane Siebourg-Polster ${ }^{4}$, Krisztina Oroszlan-Szovik ${ }^{1}$, Helga

6 Remy $^{1}$, Markus Haenggi ${ }^{1}$, Marc Stawiski ${ }^{1}$, Matthias Sehlhausen ${ }^{4}$, Pierre Maliver ${ }^{4}$, Andreas Wolfert ${ }^{5}$,

7 Thomas Emrich ${ }^{5}$, Zachary Madaj ${ }^{2}$, Martha L. Escobar Galvis ${ }^{2}$, Christoph Mueller $^{6}$, Annika

8 Herrmann $^{4}$, Patrik Brundin ${ }^{2 *}$, and Markus Britschgi ${ }^{1 *}$

$10{ }^{1}$ Roche Pharma Research and Early Development, Neuroscience Discovery, Roche Innovation Center

11 Basel, F. Hoffmann-La Roche Ltd, Grenzacherstrasse 124, Basel, Switzerland

$12{ }^{2}$ Center for Neurodegenerative Science, Van Andel Research Institute, 333 Bostwick Ave. NE, Grand

13 Rapids, MI, USA

$14{ }^{3}$ Roche Pharma Research and Early Development, pREDi, Roche Innovation Center Basel, F.

15 Hoffmann-La Roche Ltd, Grenzacherstrasse 124, Basel, Switzerland

$16{ }^{4}$ Roche Pharma Research and Early Development, Pharmaceutical Sciences, Roche Innovation

17 Center Basel, F. Hoffmann-La Roche Ltd, Grenzacherstrasse 124, Basel, Switzerland

$18{ }^{5}$ Roche Pharma Research and Early Development, Pharmaceutical Sciences, Roche Innovation Center

19 Munich, Roche Diagnostics GmbH, Nonnenwald 2, Penzberg, Germany

$20{ }^{6}$ Institute of Pathology, University of Bern, Murtenstrasse 31, Bern, Switzerland

\section{Corresponding authors:}

$24 *$ Markus Britschgi, Roche Pharma Research and Early Development, Neuroscience Discovery,

25 Roche Innovation Center Basel, F. Hoffmann-La Roche Ltd, Grenzacherstrasse 124, 4070 Basel,

26 Switzerland

27 Tel: +41616879116

28 Email: markus.britschgi@,roche.com

29

30 * Patrik Brundin, Van Andel Research Institute, 333 Bostwick Ave. NE, Grand Rapids, MI 49503,

31 USA.

32 Tel: +1616.234 .5312$

33 Email: patrik.brundin@,vai.org 


\section{Abstract}

35 Intraneuronal $\alpha$-synuclein accumulation is key in Parkinson's disease (PD) pathogenesis. The

36 pathogenic process is suggested to begin in the enteric nervous system and propagate into the brain

37 already decades before diagnosis of PD. In some patients, colitis might play a critical role in this

38 process. Here we demonstrate that patients with inflammatory bowel disease exhibit $\alpha$-synuclein

39 accumulation in the colon and that experimental colitis triggers $\alpha$-synuclein accumulation in certain

40 enteric nerves of mice. The type and degree of experimental inflammation modulates the extent of

41 colonic $\alpha$-synuclein accumulation and macrophage-related signaling limits this process. Remarkably,

42 experimental colitis at three months of age exacerbates the accumulation of aggregated phospho-

43 Serine $129 \alpha$-synuclein in the midbrain (including the substantia nigra), in 21- but not 9-month-old $\alpha$ -

44 synuclein transgenic mice. This is accompanied by loss of tyrosine hydroxylase-immunoreactive

45 nigral neurons. Our data suggest that intestinal inflammation might play a critical role in the initiation

46 and progression of PD. 
48 Parkinson's disease (PD) is a progressively debilitating neurodegenerative disease affecting $1 \%$ of the

49 population above 60 years ${ }^{1}$. Typical symptoms are motor impairments including muscle rigidity,

50 tremor, and bradykinesia. Neuropathologically, PD is hallmarked by loss of dopaminergic neurons in

51 the substantia nigra (SN), a concomitant reduction of striatal dopaminergic signaling ${ }^{2}$, and the

52 presence of intraneuronal inclusions called Lewy bodies and neurites ${ }^{3}$. Lewy pathology is enriched in

$53 \alpha$-synuclein $(\alpha \operatorname{Syn})$, a presynaptic protein that tends to aggregate and become phosphorylated under

54 pathological conditions ${ }^{2}$. Rare point mutations in $\alpha$ Syn and gene multiplications also cause familial

55 forms of PD and related neurological conditions, and certain single nucleotide polymorphisms close

56 to the $\alpha$ Syn gene (SNCA) locus are associated with increased risk for sporadic PD ${ }^{4}$. These findings

57 make $\alpha$ Syn a focal point of biomarker and drug development programs for PD.

58 Several years before the first appearance of motor symptoms, many patients exhibit a variety of non-

59 motor symptoms including constipation, sleep disorder, depression, and hyposmia ${ }^{5-7}$. Indeed, co-

60 occurrence of some of these non-motor symptoms is coupled to elevated PD risk ${ }^{8-11}$. Constipation is

61 an important non-motor feature of prodromal PD, with $28-61 \%$ of patients having exhibited

62 gastrointestinal dysfunction for several years during the prodrome ${ }^{7,10,12}$. Notably, $\alpha$ Syn-

63 immunoreactive inclusions have been found in neurons of the submucosal plexus in people with PD

$64 \quad 3,13$. Taken together, this converging evidence suggests an early involvement of the enteric nervous

65 system (ENS) in the pathogenesis of PD. Already over a decade ago, Braak and colleagues

66 hypothesized that $\alpha$ Syn-immunoreactive inclusions first appear in the ENS and then gradually engage

67 the brainstem, including the dorsal motor nucleus of the vagus nerve and midbrain areas ${ }^{3,13}$. Several

68 studies in preclinical models have demonstrated that $\alpha$ Syn pathology in the gut is associated with the

69 development of $\alpha$ Syn pathology in the brain ${ }^{14-18}$. It will be critical to determine factors that regulate

$70 \alpha$ Syn accumulation in the ENS and to understand whether the process underlying $\alpha$ Syn accumulation

71 in the gut can also lead to $\alpha$ Syn pathology in the brain.

72 Inflammation can potentially trigger $\alpha$ Syn pathology in the ENS of the gut and in the brain. A recent

73 finding in children with gastrointestinal inflammation suggests an immune regulatory function of

$74 \alpha$ Syn ${ }^{19}$. Immune pathways are indeed activated in the brain and colon of PD cases ${ }^{20,21}$. Also, several 
75 genes associated with an increased PD risk have an immune system-related function ${ }^{22}$, and it was

76 recently proposed that PD heritability is not simply due to variation in brain-specific genes, but that

77 several cell types in different tissues might be involved ${ }^{23}$. Adding further genetic evidence supporting

78 that inflammation is involved in PD pathogenesis, a genome-wide association study identified

79 common genetic pathways linking PD and autoimmune disorders ${ }^{24}$. Most prominently, LRRK2, a

80 major genetic risk factor for PD also confers increased risk for developing inflammatory bowel

81 disease (IBD) ${ }^{25}$ and is known to modulate the function of monocytes, macrophages and other immune

82 cells ${ }^{26,27}$. Intriguingly, IBD is associated with an increased risk for developing PD and specifically

83 blocking the TNF pathway reduces this risk ${ }^{28-31}$. This suggests that the intestinal immune

84 environment plays a role in triggering PD or facilitating the molecular events involved in the earliest

85 phases of the disease process ${ }^{32}$.

86 Here we tested the hypothesis that intestinal inflammation (e.g. colitis) triggers accumulation of $\alpha$ Syn

87 in the ENS and the subsequent development of $\alpha$ Syn pathology in the brain. We discovered that

88 patients with IBD exhibited increased $\alpha$ Syn accumulation in the submucosa of the colon.

89 Experimental forms of colitis in wild type and $\alpha$ Syn transgenic mice demonstrated that the type and

90 degree of inflammation regulates the amount of $\alpha$ Syn accumulation in the colon. Macrophage-related

91 signaling limited the extent of $\alpha$ Syn immunoreactivity. When $\alpha$ Syn transgenic mice were exposed to

92 experimental colitis at 3 months of age and then were aged normally up to 9 or 21 months, the

93 accumulation of aggregated $\alpha \mathrm{Syn}$ in midbrain, including the $\mathrm{SN}$, was much exacerbated in the 21-

94 months age group, but not in the 9-months age group. These 21-month old mice also exhibited loss of

95 nigral tyrosine hydroxylase-immunoreactive neurons. Together, our data support a critical role for

96 intestinal inflammation in the initiation and progression of PD. 


\section{Results}

\section{IBD patients show aSyn accumulation in the ENS and local macrophages}

99 Recent epidemiological data links inflammatory bowel disease (IBD) to an increased PD risk ${ }^{28-30}$. In

100 order to explore if IBD is associated with enteric $\alpha$ Syn accumulation we performed

101 immunohistochemistry for $\alpha$ Syn in cryo-sections from colonic biopsies of patients with ulcerative

102 colitis ( $\mathrm{UC}, \mathrm{n}=11$, mean age 31 years), Crohn's disease $(\mathrm{CD}, \mathrm{n}=11$; mean age 35 years), and from

103 healthy subjects (HS, $\mathrm{n}=8$; mean age 51 years). We observed in eight UC cases various degrees of

$104 \alpha$ Syn accumulation, mostly in structures with the morphology of neurites (Figure 1). Interestingly, the

105 eight UC cases, and four patients with CD (images not shown) also showed marked intracellular $\alpha$ Syn

106 staining in many infiltrating monocytic cells. In contrast, only one HS showed a few cells

107 immunoreactive for $\alpha$ Syn (images not shown). This finding in human tissue suggests a potential role

108 of local inflammation in the development of enteric $\alpha$ Syn accumulation.

\section{Experimental IBD exacerbates aSyn load in submucosal plexus of aSyn transgenic and wildtype}

111

112

mice

During the process of further characterizing a (Thy1)-h[A30P] $\alpha$ Syn transgenic mouse line ${ }^{33}$ we detected human $\alpha$ Syn accumulation in all innervated organs that we analyzed (Supplemental Figure 1). This included the myenteric and submucosal plexuses of the ENS, where human $\alpha$ Syn co-localized with peripherin, a specific marker for peripheral nerves (Figure 2A). We observed an age-dependent increase of baseline human $\alpha$ Syn inclusions (irregularly sized and shaped inclusion bodies detected by human $\alpha$ Syn specific monoclonal antibody clone 211) in both plexuses between the ages of three and twelve months (Figure 2B). We wanted to test whether IBD-related inflammation in the colon exacerbates this local accumulation of $\alpha$ Syn acutely (e.g. within a few days or weeks) and how the age of the $\alpha$ Syn transgenic mice influenced the outcome. Administration of dextran sulfate sodium (DSS) in the drinking water in acute or chronic paradigms are well-established mouse colitis models of IBD, exhibiting infiltration of leukocytes into the submucosa with various degrees of destruction of the colonic mucosa and submucosa ${ }^{34}$. Due to awareness of the variability of the DSS model in different genetic backgrounds of mice, we first tested DSS administration at different concentrations 
125 and durations in the (Thy1)-h[A30P] $\mathrm{S}$ Syn transgenic mice (Figure 2C), and observed leukocyte

126 infiltration in a dose-dependent manner and which was similar at the age of 3 and 6 months (Figure

127 2D and 3A). In the acute paradigm with mice at the age of 3 months, $2.5 \%$, but not $1 \%$, DSS

128 triggered intracellular accumulation of $\alpha$ Syn in nerves of the submucosal plexus (Figure 3A, B). In

129 the chronic DSS paradigm, which was done with mice at the age of 6 months, we observed a dose-

130 dependent increase of $\alpha$ Syn load in the submucosal plexus, but at a smaller magnitude than in the

131 younger mouse cohort (Figure 3A). Wildtype mice also express endogenous $\alpha$ Syn in innervated

132 organs, but at much lower levels compared with the overexpressed human $\alpha$ Syn protein in the

133 heterozygous (Thy1)-h[A30P] 2 Syn transgenic mice (Supplemental Figure 1). To confirm that the

134 finding in (Thy1)-h[A30P] Syn transgenic mice was independent of transgenic expression of human

$135 \alpha$ Syn, we applied acute and chronic (consistent dose) DSS paradigms also in wildtype mice. In both

136 treatment paradigms, we observed an elevated number of inclusion bodies of endogenous murine

$137 \alpha$ Syn in the submucosal plexus (detected by rodent cross-reactive $\alpha$ Syn-specific monoclonal antibody

138 Syn1/clone 42, Figure 3C, D). A separate experiment also confirmed that the observed effects of DSS

139 could not be attributed to increased gene expression of murine or the transgenic human $\alpha$ Syn

140 (Supplemental Figure 2). Together, these results confirmed the validity of this experimental IBD

141 paradigm to test the effect of inflammation on $\alpha$ Syn accumulation in the ENS in wild type and

142 (Thy1)-h[A30P] $\alpha$ Syn transgenic mice. Because 3-month old (Thy1)-h[A30P] $\alpha$ Syn transgenic mice

143 provided more optimal conditions for visualization and quantification of $\alpha$ Syn inclusions in the ENS,

144 for the remainder of the study we focused on using this transgenic mouse model.

146 Colitis induced by peroral DSS but not by peritoneal administration of LPS aggravates aSyn

147 accumulation in colonic submucosal plexus of aSyn transgenic mice

148 In order to explore effects of different approaches to induce inflammation in or nearby the gut in

149 (Thy 1)-h[A30P] $\alpha$ Syn transgenic mice, we compared the outcome of acute 5\% DSS in drinking water

150 with acute $0.5 \mathrm{mg} / \mathrm{kg}$ intraperitoneal LPS administration (Figure $2 \mathrm{C}$ and 4). In order to maximize the

151 inflammatory response, we administered both DSS and LPS at high, but still tolerable, doses. At day

1527 , both agents had induced variable degrees of leukocyte infiltration in the submucosa of the colon 
153 while a marked destruction of the mucosa was induced when giving only DSS (Figure 2D). As

154 before, the DSS-exposed mice presented with increased accumulation of $\alpha$ Syn in the ganglia of the

155 submucosal plexus (Figure 4A). In contrast, we detected no change in aSyn load in the myenteric

156 plexus, consistent with lack of leukocyte infiltration in this part of the colonic wall (Figure 4B).

157 Despite the high dose, LPS-induced inflammation did not increase $\alpha$ Syn accumulation in the colonic

158 nervous plexuses (Figure 4C, D). Notably, LPS and DSS resulted in a differential expression of

159 cytokines, and consistent with leukocyte recruitment, CCL2 was elevated in both (Figure 4F, G). In

160 the LPS paradigm, mRNA for IL-10 was markedly elevated, whereas DSS strongly increased IL-6

161 and also IL-1 $\beta$ but not IL-10. Together these results indicate that only certain types of local

162 inflammation increase the intracellular accumulation of $\alpha$ Syn in the colon.

\section{Lack of Cx3cr1 signaling during DSS colitis aggravates aSyn load in the submucosal plexus of}

\section{aSyn transgenic mice}

166 In both the IBD patients and the (Thy1)-h[A30P] $\alpha$ Syn transgenic mice that experienced acute DSS

167 colitis, we observed several $\alpha$ Syn-positive cells with a morphology consistent with them being

infiltrating leucocytes (Figure 5). In the mice, these infiltrating cells were positive for the

macrophage marker Iba-1 (Figure 5C-D). In order to explore further the role of

176 infiltrating leukocytes following exposure to DSS located to the mucosa and submucosa and was not

177 significantly higher in the Cx3cr1-deficient $\alpha$ Syn transgenic mice than in the Cx3cr1-competent mice

178 (Supplemental Figure 2A). However, a significantly higher level of $\alpha$ Syn accumulated in the 
181 the myenteric plexus, we found no significant increase in $\alpha$ Syn accumulation in neither the $\alpha$ Syn

182 transgenic mice with normal Cx3cr1 nor the $\alpha$ Syn transgenic mice deficient in Cx $3 \mathrm{cr} 1$, indicating

183 again a prominent role for the localization of leukocyte infiltration in the process of $\alpha$ Syn

184 accumulation in the submucosa (Figure 4B). Collectively, our results indicate a potential link

185 between monocyte/macrophage signaling and $\alpha$ Syn accumulation in ENS in this experimental IBD

186 model.

Systemic IL-10 ameliorates DSS-induced colitis and associated enteric aSyn accumulation in $\alpha$ Syn transgenic mice

190 As mentioned above, LPS-induced colonic leukocyte infiltration did not result in increased accumulation of $\alpha$ Syn in the ENS of the colon and that the main difference in cytokine expression

192 between the DSS and LPS paradigms was increased IL-10 expression in the LPS group (Figure 4).

193 Interleukin-10 is an important regulator of monocytes/macrophages, and genetic ablation of IL-10

194 signaling or blocking IL-10 with specific antibodies has been reported to enhance DSS colitis ${ }^{38,39}$. To mimic the effect of higher levels of IL-10 in an acute model of DSS colitis (5\% DSS, Figure 2C), we administered intravenously murine IL-10 (mIL10), which was recombinantly engineered onto two

197 different murine IgG variants to extend the half-life of mIL-10 in circulation (mIgG1(v1)-mIL10 and mIgG1(v2)-mIL10, respectively). As described above, DSS induced a marked increase in leukocyte infiltration and $\alpha$ Syn accumulation, and we found this to be similar in the untreated and control IgG treated group (Figure 6A, B). By contrast, both mIgG1(v1)-mIL10 and mIgG1(v2)-mIL10 significantly reduced leukocyte infiltration in mice treated with DSS ( $<<0.0001$, one-way ANOVA with Tukey HSD post-hoc analysis; Figure 6A, B). Regarding human $\alpha$ Syn in the submucosal plexus, only mIgG1(v2)-mIL10 significantly reduced the levels in DSS treated mice ( $\mathrm{p}=0.02$, one-way

204 ANOVA with Tukey HSD post-hoc analysis; Figure 6B). Interestingly, the significantly reduced $\alpha$ Syn accumulation was associated with detectable serum exposure of mIgG1(v2)-mIL10, whereas mIgG1(v1)-mIL10 was no longer detectable at the end of the in vivo phase, after 7 days (Figure 6C).

207 These results underline further an important role for the IL-10 pathway in keeping $\alpha$ Syn accumulation 208 at a reduced level throughout the course of experimental IBD. Together, our results with the genetic 
209 (e.g., CX3CR1-CX3CL1 axis) and pharmacological modulation (e.g., IL-10) of DSS colitis

210 corroborate an important role for monocyte/macrophage pathways in the development of $\alpha$ Syn

211 accumulations in the ENS of the colon.

DSS colitis-induced submucosal aSyn accumulation at a young age persists for months and is

215 IBD increases PD risk ${ }^{28-30}$ and our own data in colon samples from IBD patients (Figure 1) and 216 recent evidence in Crohn's disease ${ }^{40}$ indicate that such gut inflammatory conditions are associated

217 with $\alpha$ Syn accumulation in the ENS ${ }^{31}$. Because longer exposure to DSS mimics more closely the 218 chronic nature of IBD, we next elected to explore $\alpha$ Syn accumulation in the submucosal plexus of

219 (Thy1)-h[A30P] 2 Syn transgenic mice that were subjected to DSS colitis in a 4-week chronic

220 increasing dose paradigm. In order to allow for a full recovery from the chronic inflammation we then

221 left the mice for two months on normal drinking water and analyzed them at the age of 6 months

222 (Figure 2C). Because we also wanted to explore the effect of modulating monocytes/macrophages in

223 this chronic setting, an experimental arm with (Thy1)-h[A30P] $\alpha$ Syn transgenic mice crossed with

224 Cx3cr1-deficent mice was added. As expected, the area that is usually extensively covered by

225 leukocytes in the submucosal plexus of the acute DSS paradigm had returned to normal levels

226 following the two-month recovery period (Supplemental Figure 3A). Remarkably, however, $\alpha$ Syn

227 accumulation in the ganglia of the submucosal plexus was still almost doubled when compared to

$228 \alpha$ Syn transgenic mice that were not exposed to DSS, and this was exacerbated in $\alpha$ Syn transgenic mice deficient for Cx3cr1 (Supplemental Figure 3B). This suggests that accumulation of $\alpha$ Syn is not a transient effect or response and that improper function of monocytes/macrophages contributes to aggravation of this accumulation.

Experimental colitis-induced at a young age exacerbates $\alpha$ Syn brain pathology and dopaminergic

\section{4 neuron loss in old aSyn transgenic mice}

235 The previously highlighted hypothesis by Braak and colleagues associates $\alpha$ Syn brain pathology in

236 PD with $\alpha$ Syn pathology in the ENS earlier in life ${ }^{3,41}$. To assess development of brain $\alpha$ Syn pathology 
237 and to link it again to IBD risk, we exposed 3-month old hemizygous (Thy1)-h[A30P] $\alpha$ Syn transgenic

238 mice to DSS or normal drinking water and after 23 days on this chronic increasing dose paradigm

239 returned all mice normal drinking water for the rest of their life (Figure 2C). We chose to use the

$240 \alpha$ Syn transgenic model rather than wild type mice for this study because we knew that the model

241 exhibits some $\alpha$ Syn brain pathology that develops slowly also under baseline conditions. After aging

242 up to 9 or 21 months (i.e. mice aged for an additional 6 or 18 months after the chronic DSS paradigm

243 at the age of 3 months, respectively), we analyzed brain regions for pathological $\alpha$ Syn (proteinase K

244 resistant, pSer129- $\alpha$ Syn immunoreactive inclusions). When we examined the $\alpha$ Syn transgenic mice

245 exposed to DSS at 3 months of age, left to live only until to 9 months, we found that they exhibited

246 extremely low levels of pathological $\alpha$ Syn inclusions in the brain, similar to the levels seen in 9 month

247 old hemizygous (Thy1)-h[A30P] SSyn transgenic mice never exposed to DSS (Figure 7 and

248 Supplemental Figure 4). Similarly, twenty-one-month old hemizygous (Thy1)-h[A30P] $\alpha$ Syn

249 transgenic mice that only received water during their lifetimes showed relatively low levels of

250 pathological $\alpha$ Syn in the brain (Figure 7 and Supplemental Figure 4), which is consistent with

251 previous observations ${ }^{42}$. In marked contrast, the 21-month-old hemizygous (Thy1)-h[A30P]aSyn

252 transgenic mice that were exposed to DSS at 3 months of age presented with pSer129-positive $\alpha$ Syn

253 pathology throughout various brain regions in a much exacerbated fashion than mice that were aged

254 up to 21 months without having experienced DSS colitis at young age. The significant aggravation of

$255 \alpha$ Syn pathology also in the substantia nigra ( $\mathrm{p} \leq 0.01$ in a negative-binomial mixed-effects model

256 adjusting for multiple comparisons performed over all brain areas) was accompanied by a significant

257 loss of tyrosine hydroxylase (TH) and Nissl positive cells at 21 months of age ( $\mathrm{p} \leq 0.05$, Student's T-

258 test; Figure 8). Together, we found that DSS colitis at a young age caused an age-dependent

259 exacerbation of $\alpha$ Syn inclusion pathology and a loss of nigral dopaminergic neurons in the brains of

$260 \alpha$ Syn transgenic mice. 


\section{Discussion}

262 Currently, there is no therapy for PD available to slow or stop its progression and an obstacle in the

263 quest to develop one is that we do not understand how the disease develops ${ }^{43}$. Intraneuronal

264 accumulation of $\alpha$ Syn (i.e. Lewy bodies and neurites) is a key neuropathological hallmark and the

265 distribution of Lewy pathology in postmortem brain is used for staging in $\mathrm{PD}^{2,44}$. Accumulation of

$266 \alpha$ Syn has also been observed in the peripheral nervous system in PD, some individuals at risk of

267 developing the disease, and normal individuals ${ }^{45-47}$. Similar to this finding in people, $\alpha$ Syn-

268 immunoreactive inclusions have also been detected in the ENS of a transgenic mouse model prior to

269 changes in the brain ${ }^{48}$. Based on preclinical models and postmortem pathology in various organs

270 including the brain, it has also been suggested that $\alpha$ Syn pathology propagates temporospatially in a

271 prion-like manner ${ }^{3,44,49-51}$. However, the initial factors triggering $\alpha$ Syn aggregation are yet to be

272 established ${ }^{43}$ and the involvement of peripheral stimuli in the aggregation and pathogenic spread of

$273 \alpha$ Syn is only beginning to unravel.

274 In this study, we provide evidence that patients with IBD have increased $\alpha$ Syn accumulation in the

275 ENS (Figure 1) and that DSS colitis, i.e. an experimental IBD-like inflammation, triggers $\alpha$ Syn

276 accumulation in the ENS of wildtype mice and in a transgenic mouse model of PD (Figure 3).

277 Interestingly, in IBD patients and in the mouse model of IBD, we observed macrophages filled with

$278 \alpha$ Syn in the inflamed colonic wall (Figure 5). We found aggravation of enteric $\alpha$ Syn accumulation in

$279 \alpha$ Syn transgenic mice lacking Cx3cr1 signaling and amelioration of inflammation and enteric $\alpha$ Syn

280 load by systemic IL-10, suggesting that monocytes/macrophages modulate the process (Figure 4 and

281 6). We further observed that the aggravated $\alpha$ Syn accumulation in the ENS persisted even after two

282 months of recovery from DSS colitis and was aggravated in the absence of CXCR1 signaling,

283 indicating that the effect is not transient and mediated by monocytes/macrophages (Supplemental

284 Figure 3). Remarkably, 18 months but not 6 months post induction of DSS colitis (thus, at ages 21

285 months but not 9 months, respectively), $\alpha$ Syn transgenic mice had developed Parkinson-like brain

286 pathology (Figures 7, 8, and Supplemental Figure 4). This included elevated proteinase K resistant

287 pSer129-aSyn pathology in the midbrain, including the substantia nigra, and other brain regions and 
288 an average decrease of 30-50\% of TH- and Nissl-positive cells in the nigra. We chose to perform the

289 long-term experiments in $\alpha$ Syn transgenic rather than wild type mice. These particular $\alpha$ Syn

290 transgenic mice had previously been shown to slowly develop $\alpha$ Syn pathology in the brain ${ }^{33}$ making

291 them ideal when asking the question whether transient colonic inflammation can aggravate brain

292 pathology in a genetically predisposed animal. In future long-term studies, we plan to address whether

$293 \alpha$ Syn pathology develops also in the brains of wildtype mice if they sustain transient experimental

294 IBD at a young age. In our experiments presented here, colitis in $\alpha$ Syn transgenic mice recapitulated

295 the accumulation of enteric $\alpha$ Syn which is proposed to occur in humans several years before PD

296 diagnosis ${ }^{32}$. Additionally, the subsequent age-related development of $\alpha$ Syn pathology together with

297 the loss of nigral dopaminergic neurons in the brain of $\alpha$ Syn transgenic mice mimicked a progression

298 of the disease similar to what is considered to occur in PD.

299 We established that a mechanism by which peripheral inflammation promotes $\alpha$ Syn accumulation in

300 the colon potentially involves monocytes and macrophages. Both peroral DSS and intraperitoneal LPS

301 administration provoked strong local immune reactions resulting in leukocyte infiltration into the

302 submucosa of the colon. The region of the colon which was inflamed contains the submucosal plexus

303 and is anatomically separated from the myenteric plexus by a thick circular muscle (Figure 2). This

304 discrete localization of inflammation to the submucosa might explain why $\alpha$ Syn only accumulated in

305 the nerves of the submucosal plexus and not in the myenteric plexus of our mice given DSS. The

306 mechanism underlying how intraperitoneally administered LPS leads to submucosal leukocyte

307 infiltration probably involves the monocyte attractant chemokine CCL2 (Figure 4), but the specifics

308 remain to be clarified ${ }^{52}$. Indeed, CCL2 was upregulated in the colon of our DSS model. However, in

309 contrast to intraperitoneal LPS, where infiltrating macrophages were present in discrete patches in the

310 colonic wall, DSS-related macrophage infiltration was distributed both in small groups and larger

311 randomly distributed patches of cells across the entire colonic submucosa. Also, perorally

312 administered DSS destroys the mucosa of the colon, similar to some forms of ulcerative colitis,

313 resulting in the transient disintegration of the intestinal epithelial barrier. In our (Thy1)-h[A30P]aSyn

314 transgenic mice, the subsequent immune response to the infiltration of commensal bacteria evoked an 
315 elevated expression of cytokines such as IL-1 $\beta$ and IL-6. This upregulation was absent in the LPS

316 paradigm in which the intestinal mucosa remained intact. By acting on tight junctions, IL-1 $\beta$ and IL-6

317 can increase intestinal barrier permeability (gut leakiness), facilitating the recruitment of additional

318 immune cells to the site of the inflammation, eventually culminating in widespread immune activation

$319 \quad 53,54$. Consistent with the breach of barrier permeability in our mouse model, some PD patients exhibit

320 increased colonic cytokines such as IL-1 $\beta$, IL-6 and TNF, occurring together with increased intestinal

321 permeability ${ }^{20,55}$. In this context, it is also notable that Crohn's patients present with increased enteric

$322 \alpha$ Syn expression ${ }^{40}$ and even more striking that IBD patients on anti-TNF therapy have a reduced risk

323 of developing PD compared to IBD patients not given this treatment ${ }^{29}$. Here we demonstrate that

324 patients with IBD present with accumulation of $\alpha$ Syn in the ENS, as well as in infiltrating leukocytes

325 nearby. Notably, mucosal macrophages with intralysosomal $\alpha$ Syn content were previously described

326 in the intact human appendix ${ }^{56}$. These macrophages were in close proximity to the axonal varicosities

327 of the vermiform appendix which showed an enriched staining for $\alpha$ Syn in the mucosal plexus.

328 Furthermore, we recently found that the vermiform appendix contains aggregated and truncated $\alpha$ Syn

329 that has the propensity to seed aggregation of recombinant $\alpha$ Syn in vitro ${ }^{47}$. What could be a

330 functional role of the $\alpha$ Syn species found in abundance in the gut wall? Monomeric and oligomeric

331 aSyn species reportedly act as chemoattractants for neutrophils and monocytes, enhancing the

332 maturation of dendritic cells in the ENS ${ }^{19,57}$. With such a role in intestinal immunity, it is possible

333 that the tissue destruction induced by DSS in the present study led to release of $\alpha$ Syn, which perhaps

334 served as a chemoattractant for monocytes. The increased abundance of extracellular $\alpha$ Syn and altered

335 intestinal permeability, along with the DSS-evoked inflammatory response may have provided an

336 enabling milieu allowing further $\alpha$ Syn accumulation in the ENS of the colon ${ }^{58}$. Macrophages and

337 other immune cells are also regulated by several genes including LRRK2, an established risk gene for

338 PD and IBD. It will be interesting to explore how mutations in genes that control autophagy,

339 including the LRRK2 gene, influence the handling of $\alpha$ Syn by macrophages that invade the inflamed

340 colon in our DSS colitis paradigm. Despite the intriguing translational aspect of our finding in the

341 DSS paradigm, others have very recently reported that DSS colitis in mice down-regulates the 
342 expression of enteric $\alpha$ Syn on protein levels in vivo ${ }^{59}$. This is in contrast to our immunofluorescence

343 (e.g. increased accumulation of $\alpha$ Syn in submucosal plexus upon DSS colitis; Figures 3, 4, and 6)

344 and gene expression data (e.g., no change in endogenous and transgenic $\alpha$ Syn upon DSS colitis;

345 Supplemental Figure 2) in the same paradigm and may reflect the well-known lab-to-lab variability

346 that can occur for the DSS models ${ }^{60}$.

347 Perhaps the most striking finding in our study was that a single period of DSS-induced colitis at a

348 young age led to an exacerbation of $\alpha$ Syn pathology in the brain of $\alpha$ Syn transgenic mice much later

349 in life (Figure 7). How does the severe $\alpha$ Syn inclusion pathology develop in the brain of these mice?

350 One hypothesis is that the brain $\alpha$ Syn pathology observed in this study could be due to direct effects

351 of peripheral immune activation on the brain and that certain peripheral triggers can directly affect

352 microglial activity. For instance, short-chain fatty acids derived from gut microbiota appear to

353 influence function and maturation of microglia in the mouse brain ${ }^{61}$ and inflammatory mediators

354 released by gut microbiota into the bloodstream have been suggested to induce brain pathology and

355 behavioral changes in an $\alpha$ Syn transgenic mouse model ${ }^{62}$. Moreover, rats and nematodes have been

356 reported to develop $\alpha$ Syn inclusions after exposure to the bacterial amyloid protein curli, a protein

357 which stimulates microgliosis, astrogliosis, and secretion of IL-6 and TNF ${ }^{63}$. Intriguingly, a recent

358 study reported that peripherally applied inflammatory stimuli induce acute immune training (that

359 exacerbates $\beta$-amyloid pathology) and immune tolerance in the brain that reprograms microglia, an

360 effect which can persist for at least six months ${ }^{64}$. Whether this is a relevant mechanism in the DSS

361 paradigm needs to be explored.

362 Another hypothesis is that the brain $\alpha$ Syn pathology observed may have accumulated following the transfer of pathogenic $\alpha$ Syn seeds from the gut via the vagal nerve. Several experimental studies have demonstrated that pathogenic $\alpha$ Syn seeds can be transferred from the peripheral to the central nervous system. Aggregated recombinant $\alpha$ Syn injected intraperitoneally, intramuscularly or into the gastric wall of certain mouse models of PD results in $\alpha$ Syn inclusions in the brain ${ }^{16,65}$. Data from animals injected with $\alpha$ Syn protein in the gut wall or viral vectors expressing $\alpha$ Syn into the vagal nerve 
369 PD was also suggested by an epidemiological study indicating that vagotomy in a Danish population

370 is associated with decreased PD risk ${ }^{67}$, although this association has been challenged ${ }^{68}$. In the present

371 study, $\alpha$ Syn pathology was much more prominent in the reticular nucleus (including the vagal area)

372 and midbrain areas (compared to the rostral areas) at 18 months post DSS colitis. Although we did not

373 conduct the definitive experiment of cutting the vagal nerve, our data support the growing body of

374 evidence that the vagal nerve is involved in the accumulation of $\alpha$ Syn aggregates in the brain.

375 In summary, here we report that individuals with IBD exhibit $\alpha$ Syn accumulation in the colon

376 concomitant with infiltrating monocytes/macrophages positive for $\alpha$ Syn. We also show that $\alpha$ Syn

377 accumulates in the colon of $\alpha$ Syn transgenic and wildtype mice subjected to DSS colitis and that this

378 process is modulated by monocyte/macrophage-related signaling. We further demonstrate that chronic

379 DSS colitis in young $\alpha$ Syn transgenic mice leads to a markedly exacerbated accumulation of $\alpha$ Syn

380 aggregates in the brain when the mice age. In the same aged mice, the numbers of TH- and Nissl

381 positive neurons in the substantia nigra are reduced, suggestive of a neurodegenerative process.

382 Together, our findings are in consonance with studies demonstrating a link between IBD and PD

$38328,29,69$ and suggest a critical role for intestinal inflammation and $\alpha$ Syn accumulation in the initiation

384 and progression of $\mathrm{PD}$. 


\section{Methods}

\section{Mice}

387 Male C57BL/6 wild type mice (Jackson Laboratories, Bar Harbor, USA), hemizygous Tg(Thy1-

388 SNCA*A30P)18Pjk ((Thy1)-h[A30P] 2 Syn $)^{33}$ and Tg(Thy1-SNCA*A30P)18Pjk crossed with

389 Cx3cr1tm1Litt ((Thy1)-h[A30P] $\alpha$ Syn /CX3CR1-def; homozygous for Cx3cr1-GFP knock-in allele; ${ }^{70}$

390 transgenic mice were used for the study. (Thy1)-h[A30P] $\alpha$ Syn transgenic mice express mutant human

$391 \alpha$ Syn under the neuron selective Thy1 promoter. (Thy1)-h[A30P] $\alpha$ Syn transgenic mice were crossed

392 to Cx3cr1-def transgenic mice which express eGFP replacing fractalkine gene expression. All mice

393 were maintained on a C57BL/6 background for more than 10 generations and under specific

394 pathogen-free conditions. To the extent possible, littermates were used in the experiments. Health

395 status was monitored daily during experiments.

\section{Human Subjects}

398 Samples from patients with Crohn's disease (CD), ulcerative colitis (UC) or healthy subjects (HS)

399 were provided by the tissue bank, Institute of Pathology, University of Bern. Briefly, specimens were

400 obtained from patients who underwent surgical procedures at the University Hospital (Inselspital) in

401 Bern, Switzerland between 2004 and 2011. Three selected male patients previously clinically diagnosed with $\mathrm{UC}$ with a reported disease duration $>6$ year $(\mathrm{n}=3)$ and undergoing steroid therapy combined with either metronidazole or mesalazine. CD patients were of mixed gender and aged 22-56

404 years ranging from 2 months to 11 years post disease diagnosis undergoing treatment with either infliximab or mesalazine in combination with steroids. Healthy subjects were of mixed gender with no report of inflammatory bowel disease, aged 40-59. All samples contained the mucosa and submucosa regions including minor parts of the circular muscle layer. Following surgical removal, tissue samples were immediately immersed in O.C.T. compound (VWR International GmbH, Dietikon, Switzerland),

409 frozen in liquid nitrogen and stored at $-80^{\circ} \mathrm{C}$. Diagnosis of disease status was made according to 410 established criteria for histopathological analysis. 


\section{Experimental IBD in mice with DSS and LPS}

413 Paradigms for the induction of inflammation were either 1 week (acute) or 3-4 weeks (chronic) with

414 or without an incubation phase of 2, 6 or 18 months post application (Figure 2). Acute systemic

415 inflammation was induced by intraperitoneal Lipopolysaccharide (LPS) application ${ }^{71}$ of $0.5 \mathrm{mg} / \mathrm{kg}$ in

$416100 \mu 1$ injection volume on day 1 and 4 (Sigma-Aldrich Chemie GmbH, Steinheim, Germany, LPS

417 055:B5). Acute colitis was induced by application of 36-50kDa Dextran Sulfate Sodium (DSS) ${ }^{72}$

418 (160110, MP Biomedicals, LLC, Illkirch, France) at $0 \%, 1 \%, 2.5 \%$ or $5 \%$ in autoclaved drinking

419 water for 5 continuous days respectively, followed by 2 days of water (1 DSS application cycle).

420 Chronic colitis was achieved by 4 repeating DSS application cycles. The DSS concentration during 4

421 weeks of chronic colitis was either $1 \%$ or $2.5 \%$ for 4 weeks or $2.5 \%-4 \%$ raised $0.5 \%$ every week for 4

422 weeks. Mice from same littermate group were randomized per cage into vehicle and inflammation

423 inducing agent.

\section{IL-10 treatment and exposure measurement}

426 Two different forms of mouse IgG bound murine IL-10 (mIgG(v1)-mIL10 and mIgG(v2)-mIL10)

427 were diluted in pre-prepared sterile formulation buffer comprised of $0.5 \%$ mouse serum supplemented 428 with $25 \mathrm{mM}$ citrate, $300 \mathrm{mM}$ arginine to a final concentration of $0.75 \mathrm{mg} / \mathrm{ml}$ and the $\mathrm{pH}$ adjusted to 6.7

429 on the day of application. Each mouse was treated once with $150 \mu \mathrm{g}$ i.p concurrently with the

430 initiation of the acute colitis paradigm with 5\% DSS. The concentrations of mIgG-mIL10 fusion

431 proteins in murine serum samples were determined by enzyme-linked immunosorbent assays (ELISA)

432 specific for the Fab moiety of the administered mIgG-mIL10 fusion protein. Biotinylated mIgG-

433 mIL10-specific target molecules were used for capturing, goat anti-mIg IgG-HRP conjugate and

434 peroxidase substrate ABTS was used for quantitative detection of $\mathrm{mIgG}-\mathrm{mIL} 10$ fusion proteins.

Immunohistochemistry

437 Animals were injected with a lethal dose of pentobarbital $(150 \mathrm{mg} / \mathrm{kg})$. Upon full anesthesia, mice

438 received transcardial perfusion with room temperature phosphate buffered saline (PBS). For

439 biochemical and immunohistochemical analysis, one section of either the proximal colon was fresh 
440 frozen and stored at $-80^{\circ} \mathrm{C}$ or post-fixed in $4 \%$ paraformaldehyde (PFA) solution for $24 \mathrm{~h}$. Following

441 post-fixation, organs were incubated in $30 \%$ sucrose/PBS at $4{ }^{\circ} \mathrm{C}$ for at least $48 \mathrm{~h}$ before further

442 processing. Subsequently, enteric tissue was cryotome-sectioned to $35 \mu \mathrm{m}$ thick longitudinal sections

443 (approx. $1 \mathrm{~cm}$ length). The brain was collected and post-fixed for $24 \mathrm{~h}$ in $4 \%$ PFA followed by $30 \%$

444 sucrose in phosphate buffer until cryo-sectioning of floating sections at $40 \mu \mathrm{m}$. Histological analysis

445 of the colon was performed using standard hematoxylin staining. Immunohistochemical staining was

446 accomplished using the Vectastain Elite ABC Kits and Peroxidase Substrate Kit SK-4100 (Vector

447 Laboratories, Burlingame, CA, USA) or fluorescently labelled secondary antibodies (Alexa coupled

448 to dye 488,555 or 647 , Life Technologies, Zug, Switzerland). The following primary antibodies have

449 been used for overnight incubation at a dilution of 1:1000: monoclonal antibody to human $\alpha$-synuclein

450 (clone 211, sc-12767, Santa Cruz Biotechnology, Heidelberg, Germany; used on tissue from human

$451 \alpha$ Syn transgenic mice), monoclonal antibody to rat $\alpha$-synuclein but cross-reactive with murine and

452 human $\alpha$ Syn (Syn1/clone 42, BD Transduction Laboratories, Allschwil, Switzerland; used for wild

453 type mice and in human colon), polyclonal antibody to the peripheral neuronal marker Peripherin

454 (Millipore Corporation, Billerica, MA, USA), and polyclonal antibody to macrophage marker Iba1

455 (Wako Chemical GmbH, Neuss, Germany). To detect phosphorylated $\alpha$ Syn (pSer129 pathology) in

456 the free-floating brain sections, monoclonal antibody (ab51253, Abcam, Cambridge, USA) to human

$457 \alpha$ Syn was used at a dilution of 1:10000. Prior to the pSer129 staining, the free-floating brain sections

458 were incubated for $10 \mathrm{~min}$ at room temperature in a phosphate buffered saline solution containing 10

$459 \mu \mathrm{g} / \mathrm{mL}$ proteinase K (Cat \# 25530015; Invitrogen, California, USA). TH-immunoreactive cells were

460 detected using a polyclonal antibody (657012, Millipore Sigma) at a dilution of 1:1000. To measure

461 the density of Nissl-positive cells, the TH-stained cells were counter-stained with Cresyl violet. The

462 slides were incubated in $0.1 \%$ Cresyl violet solution for 9 min and then dehydrated in $95 \%$ and $100 \%$

463 ethanol and then xylene prior to coverslipping with Cytoseal 60 mounting media (Thermo Fisher

464 Scientific). Quantifications of the blind-coded TH/Nissl stained slides were done using

465 Stereoinvestigator (version 2017.01.1; MBF Bioscience, Williams, VT, USA) on Imager M2

466 microscope (ZEISS) coupled to a computer. We analyzed 5-7 nigral sections per animal, and a total of

$467 \quad 7-8$ animals per treatment group. We outlined the substantia nigra pars compacta and counted every 
468 TH-immunoreactive and Nissl-positive cell in that area and computed the number of cells per section,

469 generating the mean cell density per animal. We then calculated the mean density of cells per

470 treatment group and analyzed the data using unpaired Student's T-test after confirming normality and

471 homoscedasticity in Prism 7.0 (GraphPad Software).

472

473 Imaging and stereological quantification of aSyn deposits in enteric nervous system

474 Imaging and stereological quantification was performed on a Zeiss Axio Imager Z2 fluorescence

475 microscope (Carl Zeiss AG, Jena, Germany). Leica TCS SP5 confocal system using an HCX PL APO

476 CS 40x 1.3 oil UV or an HCX PL APO LB 63x 1.4 oil UV objective was utilized for image recording.

477 Accumulation of $\alpha$ Syn in the ENS was assessed on a random set of 3 adjacent $35 \mu \mathrm{m}$ thick, $\alpha$ Syn-

478 immunostained sections comprising the myenteric and submucosal neuronal plexuses. Analysis was

479 performed with the aid of Stereologer software (Stereo Investigator 10, MBF Bioscience, Williams,

$480 \mathrm{VT}, \mathrm{USA}$ ) as described previously ${ }^{73}$. In the myenteric plexus ganglion volume was defined by

481 multiple outlined plexuses containing a range of 5-20 neuronal cells and quantified by the optical

482 fraction fractionator technique. In contrast to the myenteric plexus, the submucosa consists of

483 compact plexuses with 1-5 cells including interconnecting neurites. Therefore, the entire submucosa

484 was set as region of interest, analyzed with the area fraction fractionator technique. Results of the

485 submucosal plexus are displayed by percent area containing aSyn deposits. For the IL-10 experiment,

486 aSyn positive inclusions from immunofluorescence images were counted for each image. Inclusion

487 body-like features were filtered based on having a size between 12 and 50000 pixels and a minimal intensity value greater than 300 . The filtering step was included to exclude small background features and macrophages (very large spots). The counts were then aggregated to the animal level by summing the inclusion feature counts of all images per animal and then normalizing for (i.e. dividing by) the number of images for a given animal. Upon exploratory data analysis two animals were excluded: one mouse because it only had one image and another due it being an outlier, based on its infiltration score and image data.

\section{Blinding of experimenters}


496 For analyses of colon and brain tissue on slides, a second individual assigned unique codes to

497 stained slides. Therefore, the experimenter conducted the analyses blinded to the identity of the

498 mice. For randomization of treatment groups see above.

Quantification of leukocytes infiltration

501 To determine the leukocyte covered area in the colon after LPS or DSS application, three adjacent

502 hematoxylin stained sections were quantified. Total area of colon sections and localizations of

503 leukocyte assemblies within the tissue architecture were identified and outlined utilizing Stereologer

504 Software (Stereo Investigator 6, MBF Bioscience, Williams, VT, USA). Percentage of leukocyte

505 covered area has been set in proportion to total area of the analyzed colon section. For the IL-10

506 experiment, hematoxilin stained colon slices were examined by an expert pathologist blinded to

507 treatment conditions. A score of 0-3 was assigned to each section for each of the 3 layers lamina

508 propria, submucosa and muscularis based on the degree of inflammatory infiltration. A score of 0

509 denoted no inflammation and a score of 3 indicated extensive infiltration. The mean of the values for

510 all 3 layers was taken as the final measure of leukocyte infiltration per mouse.

511

\section{Quantification of aSyn/Iba1 double positive macrophages}

513 The number of $\alpha$-syn+/Iba1+ positive cells was evaluated by quantification of 10 random regions in 2

514 adjacent sections of the proximal colon. The region of interest was set to contain the myenteric

515 plexus/circular muscle layer and the submucosal plexus. Cells were assessed for positive $\alpha$ Syn

516 staining and concomitant co-localization with the macrophage marker Iba1 was quantified.

\section{Scoring of pSer129 pathology and brain heatmap}

519 We evaluated pSer129 pathology on a full series of immunostained coronal sections from 10 mice per 520 treatment group (i.e. water vs. DSS-treated groups) on blind-coded slides using a previously described

521 method $^{74}$. We visualized pathology from one hemisphere of all brain sections (apart from the

522 olfactory area) using NIKON Eclipse Ni-U microscope and assigned scores ranging from 0 to 4 to 
523 each brain area based on the relative abundance of PK-resistant pSer129-positive inclusions (i.e. cell

524 bodies and neurites). In this case, $0=$ no aggregates, $1=$ sparse, $2=$ mild, $3=$ dense, $4=$ very dense.

525 For the heatmap, we obtained the average score values of each brain area for each treatment group.

526 The average data for each treatment group ( $\mathrm{n}=10$ / group) was then represented as a heatmap in a

527 sagittal mouse brain background (http://atlas.brain-

528 map.org/atlas?atlas $=2$ \#atlas $=2 \&$ structure $=771 \&$ resolution $=16.75 \& \mathrm{x}=$

$529 \quad \underline{7755.7470703125 \& \mathrm{y}=3899.625 \& \mathrm{zoom}=-3 \& \text { plate }=100883867 \& \mathrm{z}=5)}$.

Densitometry of pSer129 aSyn brain pathology

532 The density of pSer129 pathology in 12 major brain areas (reticular nucleus, pontine reticular nucleus,

533 periaqueductal gray, gray and white layer, reticular formation, substantia nigra, ventral tegmental

534 area, thalamus, hypothalamus, central amygdala, pallidum and striatum) was determined in the water

535 and DSS-treated animals. A NIKON Eclipse Ni-U microscope was used to acquire 20x magnification

536 images (without condenser lens) from all the indicated brain areas, using the same exposure time for

537 all images. In all cases, images were acquired on three sections separated by $420 \mu \mathrm{m}$ intervals

538 (localized between Bregma). We then processed the acquired images using Image J64 ${ }^{75}$, created a

539 mask (to exclude background) that redirects to the original image for analysis, measured the total area

540 and the mean grey value of the area that had inclusions. For brain areas such as periaqueductal gray

541 that do not fill the entirety of the field to be analyzed, we drew a contour of the area and the analysis

542 was performed only within that contoured area. We subsequently calculated the grey value of the area

543 per square pixels for each image (i.e. A.U. $/ \mathrm{px}^{2}=$ mean grey value $\mathrm{x}$ area stained/total area assessed).

544 Based on this, we calculated the average grey value per square pixels for each brain area for each

545 animal ( $n=6$ mice/group), and then extended this calculation to determine the average grey value per

546 square pixels for each treatment group and each of the twelve brain areas of interest.

mRNA expression

549 To assess mRNA expression levels from the proximal colon, RNA was extracted from fresh frozen

550 tissue with MagnaLyser green beads (Roche Diagnostics, Mannheim, Germany) and Qiazol Lysis 
551 (Reagent cat.no.79306, Hilden, Germany) purified on MagnaPure LC (HP Kit no.03542394001, F.

552 Hoffmann - La Roche AG, Rotkreuz, Switzerland) and amplified via real-time PCR (4ng

553 RNA/reaction; Lightcycler 480, Roche Diagnostics Corporation, Indianapolis, USA). Amplification

554 of mRNA was performed by using TaqMan probes for human or murine specific $\alpha$-synuclein and for

555 selected cytokines/chemokines (Applied Biosystems Europe B.V., Zug, Switzerland). Target mRNA

556 was normalized to tissue-specific murine GAPDH levels and displayed as relative expression after 30

557 amplification cycles.

Statistics

560 Statistical analysis of gut pathology and inflammation was performed using GraphPad Prism 6.04 or

5617.0 software (GraphPad Software, Inc. La Jolla, CA, USA). The results are expressed as mean values

$562 \pm$ standard errors of the mean (SEM). Student's T-test (or Welch's T-test for unequal variances) was

563 used to compare two groups and ANOVA was used for multi-comparison of groups followed by

564 Tukey HSD post-hoc analysis. For the statistical analysis of the pSer129 $\alpha$ Syn brain pathology,

565 negative-binomial mixed-effects models with a random intercept for each sample were used to

566 analyze the dataset via the 'Ime4' (http://lme4.r-forge.r-project.org/) package in R v 3.4.4. To analyze

567 the pSer129 $\alpha$ Syn cell count dataset, an offset for the total area examined was included to model the

568 densities. Linear contrasts with false discovery rate (FDR) adjustments were then used to test our

569 hypotheses and account for multiple testing (for brain area and experimental group). Like the pSer129

570 dataset, the Iba-1/aSyn-double positive dataset were analyzed using negative-binomial regression and

571 Tukey HSD adjusted contrasts to test our hypotheses.

573 For the statistical analysis of the mRNA expression, data quality was assessed by inspecting the

574 distribution of $\mathrm{Cp}$ values of reference endogenous genes across samples, by inspecting the level of $\mathrm{Cp}$

575 variation between technical replicates and by exploring the samples multivariate signal distribution as

576 in a principal component analysis. Relative gene expression levels were expressed as $2^{-(\mathrm{Cpgene}-\mathrm{CpRef})}$.

577 Statistical analyses to assess the effect of the experimental conditions on the log2 gene expression 
578 levels were done with linear models using the limma package (Bioconductor/R, Smyth, 2005). These

579 analyses were implemented in R v3.1.1.

581 For the statistical modelling of the effects of the IL-10 treatment on $\alpha$ Syn counts, as well as

582 infiltration scores, the levels for IgG1(v1)-IL10 and IgG1(v2)-IL10 treatment were compared to the

583 positive (vehicle/DSS) control. Additionally, since levels of the control antibody treatment (IgG1(v1))

584 were very similar to the positive control, the two groups were pooled in further contrasts in which

585 effects of individual antibodies or control IgG was assessed. For $\alpha$ Syn counts, a linear model on the

586 treatment groups with one-degree freedom contrasts was applied. For the infiltration score a Kruskal-

587 Wallis test, with the same contrasts, was used.

\section{Study approvals}

590 The human subjects' study was conducted with the approval of the local Ethical Committee in Bern

591 No. 47/04. Written informed consent was obtained from each patient. The animal experiments were approved by a Roche internal review board and the local authorities.

\section{Author contributions}

596 S.G., N.M. and L.S. planned and performed the in vivo experiments, colon immunostaining, analysis, 597 and quantification; S.G. and N.M. drafted a first version of the manuscript; E.Q. performed, imaged, quantitated pSer129, TH and Nissl staining in the brain sections, and drafted a more advanced version

599 of the manuscript with J.A.S., who also provided helpful discussion. F.B. and K.O.S. supported the

600 image acquisition and image analysis for the colon samples; M.St. performed imaging and data

601 analysis of experiments with wildtype mice; G.D.P. and J.S.P. performed statistical analysis of the

602 DSS experiments; H.R. and M.H. performed mRNA analyses; M.Se. trained S.G. and L.S. on mouse

603 necropsy and supported their work; P.M. performed expert pathology staging on leukocyte

604 infiltration; T.E. and A.W. provided mIgG-mIL-10 fusion proteins and measured serum exposure;

605 Z.M. performed statistical analysis for the pSer129 $\alpha$ Syn immunohistochemistry data. M.L.E.G. 
606 provided helpful discussion and project planning. A.H. co-mentored S.G. and N.M., performed expert

607 pathology staging on leukocyte infiltration and contributed to experimental planning. C.M. trained

608 S.G. on the colitis model, provided human tissue and expert input on the experimental IBD model.

609 M.B. and P.B. co-mentored Roche Postdoctoral Fellows S.G. and N.M., conceived and oversaw the

610 study, and performed experimental planning; M.B., P.B. and E.Q. wrote the final version of the

611 manuscript.

612

613

\section{Acknowledgments}

615 We acknowledge the human donors for providing tissue used in this study. We thank Drs. L. Ozmen,

616 A. Bergadano, and A. Su for their tremendous support in maintaining the mouse colony and

617 establishing of relevant animal experiment licenses, and we are grateful to the animal care takers,

618 veterinarians and many unnamed staff at Roche for their valuable work with the mice in this study. In

619 addition, at Roche we thank Dr. K.G. Lassen for critical input to the paper, Dr. C. Ullmer for co-

620 mentoring S.G. and providing scientific input, Dr. L. Collin for helping with confocal imaging and we

621 are grateful to Dr. T. Kremer, N. Haenggi, D. Mona, A. Girardeau, and J. Messer for providing

622 support in tissue dissections and G. Walker and R. Lauria for technical support. Ms. E. Schulz from

623 VARI assisted with immunostaining of the brain tissue. We thank the Contract Research Organization

624 Frimorfo for carefully sectioning the brains for this study. We acknowledge Drs. L. Gaudimier (née

625 Chicha) and F. Pan-Montojo for scientific discussions early in the project and Dr. W. Zago from

626 Prothena for valuable scientific input throughout the project. P.B. reports relevant grants from NIH

627 (R01DC016519-01, 1R21NS106078-01A1 and 5R21NS093993-02), Department of Defense

628 (W81XWH-17-1-0534), The Michael J. Fox Foundation for Parkinson's Research, and Cure

629 Parkinson's Trust. Finally, we thank the Roche Postdoctoral Fellowship Program for supporting S.G.

630 and N.M.

631

632

633 


\section{Conflicts of Interest}

635 At the time of the study S.G. and N.M. were Roche Postdoctoral Fellows employed by Roche and

636 L.S., F.B., G.D.P., J.S.P., K.O.S., H.R., M.H., M.Se. M.St., P.M., A.W., T.E., A.H. and M.B. are or

637 were fulltime employees or trainees at Roche and they may additionally hold Roche stock/stock

638 options. S.G. and L.S. are currently employees of Neurimmune AG, Schlieren, Switzerland. P.B. has

639 received commercial support as a consultant from Renovo Neural, Roche, Teva, Lundbeck A/S,

640 AbbVie, NeuroDerm, Fujifilm Cellular Dynamics, Living Cell Technologies, IOS Press Partners, and

641 Axial Biotherapeutics. Additionally, P.B. has received commercial support as a consultant from

642 Renovo Neural, Roche, Teva, Lundbeck A/S, AbbVie, NeuroDerm, Fujifilm Cellular Dynamics,

643 Living Cell Technologies, IOS Press Partners, Axial Biotherapeutics and CuraSen. P.B. has received

644 commercial support for grants/research from Renovo, Roche, Teva, and Lundbeck and has ownership

645 interests in AcouSort AB. The other authors do not have conflicts of interest with regard to this

646 research. 


\section{References}

648 1. Tysnes, O.-B. \& Storstein, A. Epidemiology of Parkinson's disease. J Neural Transm (Vienna)

$649 \quad \mathbf{1 2 4}, 901-905(2017)$.

650 2. Spillantini, M. G. \& Goedert, M. Neurodegeneration and the ordered assembly of $\alpha$-synuclein.

$651 \quad$ Cell Tissue Res. 373, 137-148 (2017).

652 3. Del Tredici, K. \& Braak, H. Lewy pathology and neurodegeneration in premotor Parkinson's disease. Mov. Disord. 27, 597-607 (2012).

654 4. Polymeropoulos, M. H. et al. Mutation in the alpha-synuclein gene identified in families with 655 Parkinson's disease. Science 276, 2045-2047 (1997).

656 5. Schrag, A., Horsfall, L., Walters, K., Noyce, A. \& Petersen, I. Prediagnostic presentations of 657 Parkinson's disease in primary care: a case-control study. Lancet Neurol 14, 57-64 (2015).

658 6. Gaenslen, A., Swid, I., Liepelt-Scarfone, I., Godau, J. \& Berg, D. The patients' perception of prodromal symptoms before the initial diagnosis of Parkinson's disease. Mov. Disord. 26, 653$658(2011)$.

7. Pont-Sunyer, C. et al. The onset of nonmotor symptoms in Parkinson's disease (the ONSET PD study). Mov. Disord. 30, 229-237 (2015).

8. Berg, D. et al. The PRIPS study: screening battery for subjects at risk for Parkinson's disease. Eur. J. Neurol. 20, 102-108 (2013).

9. Postuma, R. B., Gagnon, J.-F., Bertrand, J.-A., Génier Marchand, D. \& Montplaisir, J. Y. Parkinson risk in idiopathic REM sleep behavior disorder: preparing for neuroprotective trials. Neurology 84, 1104-1113 (2015).

10. Abbott, R. D. et al. Frequency of bowel movements and the future risk of Parkinson's disease. Neurology 57, 456-462 (2001).

670 11. Savica, R. et al. Medical records documentation of constipation preceding Parkinson disease: A 671 case-control study. Neurology 73, 1752-1758 (2009).

672 12. Mahlknecht, P., Seppi, K. \& Poewe, W. The Concept of Prodromal Parkinson's Disease. J 
674 13. Braak, H., de Vos, R. A. I., Bohl, J. \& Del Tredici, K. Gastric alpha-synuclein immunoreactive

675 inclusions in Meissner's and Auerbach's plexuses in cases staged for Parkinson's disease-related

676 brain pathology. Neurosci. Lett. 396, 67-72 (2006).

677 14. Phillips, R. J., Walter, G. C., Wilder, S. L., Baronowsky, E. A. \& Powley, T. L. Alpha-synucleinimmunopositive myenteric neurons and vagal preganglionic terminals: autonomic pathway implicated in Parkinson's disease? Neuroscience 153, 733-750 (2008).

15. Holmqvist, S. et al. Direct evidence of Parkinson pathology spread from the gastrointestinal tract to the brain in rats. Acta Neuropathol 128, 805-820 (2014).

16. Breid, S. et al. Neuroinvasion of $\alpha$-Synuclein Prionoids after Intraperitoneal and Intraglossal Inoculation. J. Virol. 90, 9182-9193 (2016).

17. Sargent, D. et al. 'Prion-like' propagation of the synucleinopathy of M83 transgenic mice depends on the mouse genotype and type of inoculum. J. Neurochem. 143, 126-135 (2017).

18. Manfredsson, F. P. et al. Induction of alpha-synuclein pathology in the enteric nervous system of the rat and non-human primate results in gastrointestinal dysmotility and transient CNS pathology. Neurobiol. Dis. 112, 106-118 (2018).

19. Stolzenberg, E. et al. A Role for Neuronal Alpha-Synuclein in Gastrointestinal Immunity. JIN 9,

20. Devos, D. et al. Colonic inflammation in Parkinson's disease. Neurobiol. Dis. 50, 42-48 (2013).

21. Mogi, M. et al. Interleukin-1 beta, interleukin-6, epidermal growth factor and transforming growth factor-alpha are elevated in the brain from parkinsonian patients. Neurosci. Lett. 180,

22. Brás, J., Guerreiro, R. \& Hardy, J. SnapShot: Genetics of Parkinson’s disease. Cell 160, 570570.e1 (2015).

23. Reynolds, R. H. et al. Moving beyond neurons: the role of cell type-specific gene regulation in Parkinson's disease heritability. npj Parkinson's Disease 5, 6 (2019). Diseases. JAMA Neurol 74, 780-792 (2017). 
701 25. Umeno, J. et al. Meta-analysis of published studies identified eight additional common

702 susceptibility loci for Crohn's disease and ulcerative colitis. Inflamm. Bowel Dis. 17, 2407-2415

$703 \quad(2011)$.

704 26. Gardet, A. et al. LRRK2 is involved in the IFN-gamma response and host response to pathogens.

J. Immunol. 185, 5577-5585 (2010).

27. Hakimi, M. et al. Parkinson's disease-linked LRRK2 is expressed in circulating and tissue immune cells and upregulated following recognition of microbial structures. J Neural Transm (Vienna) 118, 795-808 (2011).

28. Lin, J.-C., Lin, C.-S., Hsu, C.-W., Lin, C.-L. \& Kao, C.-H. Association Between Parkinson's Disease and Inflammatory Bowel Disease: a Nationwide Taiwanese Retrospective Cohort Study. Inflamm. Bowel Dis. 22, 1049-1055 (2016).

29. Peter, I. et al. Anti-Tumor Necrosis Factor Therapy and Incidence of Parkinson Disease Among Patients With Inflammatory Bowel Disease. JAMA Neurol 75, 939-946 (2018).

30. Wan, Q.-Y., Zhao, R. \& Wu, X.-T. Older patients with IBD might have higher risk of Parkinson's disease. Gut gutjnl-2018-317103 (2018). doi:10.1136/gutjnl-2018-317103

31. Rolli-Derkinderen, M. et al. Is Parkinson's disease a chronic low-grade inflammatory bowel disease? J Neurol 1-7 (2019). doi:10.1007/s00415-019-09321-0

32. Houser, M. C. \& Tansey, M. G. The gut-brain axis: is intestinal inflammation a silent driver of Parkinson's disease pathogenesis? npj Parkinson's Disease 3, 3 (2017).

33. Kahle, P. J. et al. Subcellular localization of wild-type and Parkinson's disease-associated mutant alpha -synuclein in human and transgenic mouse brain. J. Neurosci. 20, 6365-6373 (2000).

34. Chassaing, B., Aitken, J. D., Malleshappa, M. \& Vijay-Kumar, M. Dextran sulfate sodium (DSS)induced colitis in mice. Curr Protoc Immunol 104, Unit 15.25. (2014).

35. Weber, B., Saurer, L., Schenk, M., Dickgreber, N. \& Mueller, C. CX3CR1 defines functionally distinct intestinal mononuclear phagocyte subsets which maintain their respective functions during homeostatic and inflammatory conditions. Eur. J. Immunol. 41, 773-779 (2011). translocation, and colitogenic Th17 responses in mice. J. Clin. Invest. 121, 4787-4795 (2011). 
729 37. Kostadinova, F. I. et al. Crucial involvement of the CX3CR1-CX3CL1 axis in dextran sulfate 730 sodium-mediated acute colitis in mice. J. Leukoc. Biol. 88, 133-143 (2010).

731 38. Kang, S. et al. Intestinal epithelial cell-derived semaphorin 7A negatively regulates development of colitis via $\alpha v \beta 1$ integrin. J. Immunol. 188, 1108-1116 (2012).

39. Li, B., Alli, R., Vogel, P. \& Geiger, T. L. IL-10 modulates DSS-induced colitis through a macrophage-ROS-NO axis. Mucosal Immunol 7, 869-878 (2014).

40. Prigent, A. et al. Enteric alpha-synuclein expression is increased in Crohn's disease. Acta Neuropathol 137, 359-361 (2019).

41. Braak, H. et al. Staging of brain pathology related to sporadic Parkinson's disease. Neurobiol. Aging 24, 197-211 (2003).

42. Neumann, M. et al. Misfolded proteinase K-resistant hyperphosphorylated alpha-synuclein in aged transgenic mice with locomotor deterioration and in human alpha-synucleinopathies. J. Clin. Invest. 110, 1429-1439 (2002).

43. Johnson, M. E., Stecher, B., Labrie, V., Brundin, L. \& Brundin, P. Triggers, Facilitators, and Aggravators: Redefining Parkinson's Disease Pathogenesis. Trends Neurosci. S0166-2236, 30253-30254 (2018).

44. Braak, H. et al. Staging of the intracerebral inclusion body pathology associated with idiopathic Parkinson's disease (preclinical and clinical stages). J. Neurol. 249 Suppl 3, III/1-5 (2002).

45. Shannon, K. M., Keshavarzian, A., Dodiya, H. B., Jakate, S. \& Kordower, J. H. Is alphasynuclein in the colon a biomarker for premotor Parkinson's disease? Evidence from 3 cases. Mov. Disord. 27, 716-719 (2012).

46. Lebouvier, T. et al. Colonic biopsies to assess the neuropathology of Parkinson's disease and its relationship with symptoms. PLOS ONE 5, e12728 (2010).

47. Killinger, B. A. et al. The vermiform appendix impacts the risk of developing Parkinson's disease. Sci Transl Med 10, eaar5280 (2018). chromosomes containing Parkinson disease-associated $\alpha$-synuclein gene mutations precede central nervous system changes. Hum Mol Genet 19, 1633-1650 (2010). 
757 49. Spillantini, M. G. et al. Alpha-synuclein in Lewy bodies. Nature 388, 839-840 (1997).

758 50. Luk, K. C. et al. Pathological $\alpha$-synuclein transmission initiates Parkinson-like neurodegeneration 759 in nontransgenic mice. Science 338, 949-953 (2012).

760 51. Rey, N. L. et al. Widespread transneuronal propagation of $\alpha$-synucleinopathy triggered in olfactory bulb mimics prodromal Parkinson's disease. J Exp Med 213, 1759-1778 (2016).

52. Puntambekar, S. S. et al. LPS-induced CCL2 expression and macrophage influx into the murine central nervous system is polyamine-dependent. Brain Behav. Immun. 25, 629-639 (2011).

53. Al-Sadi, R. M. \& Ma, T. Y. IL-1beta causes an increase in intestinal epithelial tight junction permeability. J. Immunol. 178, 4641-4649 (2007).

54. Capaldo, C. T. \& Nusrat, A. Cytokine regulation of tight junctions. Biochim Biophys Acta 1788, 864-871 (2009).

55. Forsyth, C. B. et al. Increased Intestinal Permeability Correlates with Sigmoid Mucosa alphaSynuclein Staining and Endotoxin Exposure Markers in Early Parkinson's Disease. PLOS ONE 6, e28032 (2011)

56. Gray, M. T., Munoz, D. G., Gray, D. A., Schlossmacher, M. G. \& Woulfe, J. M. Alpha-synuclein in the appendiceal mucosa of neurologically intact subjects. Mov. Disord. 29, 991-998 (2014). Synuclein during Gastrointestinal Infection. JIN 9, 437-440 (2017).

58. Kelly, L. P. et al. Progression of Intestinal Permeability Changes and Alpha-Synuclein Expression in a Mouse Model of Parkinson's Disease. Mov Disord 29, 999-1009 (2014).

59. Prigent, A. et al. Acute inflammation down-regulates alpha-synuclein expression in enteric CNS. Nat. Neurosci. 18, 965-977 (2015). 
785 63. Chen, S. G. et al. Exposure to the Functional Bacterial Amyloid Protein Curli Enhances Alpha-

786 Synuclein Aggregation in Aged Fischer 344 Rats and Caenorhabditis elegans. Scientific Reports

$787 \quad 6,34477(2016)$.

788

789

790

791

792

793

794

795

796

797

798

799

800

801

802

803

804

805

806

807

808

809

810

811

812

64. Wendeln, A.-C. et al. Innate immune memory in the brain shapes neurological disease hallmarks. Nature 556, 332-338 (2018).

65. Sacino, A. N. et al. Intramuscular injection of $\alpha$-synuclein induces CNS $\alpha$-synuclein pathology and a rapid-onset motor phenotype in transgenic mice. Proc Natl Acad Sci U S A 111, 10732 10737 (2014).

66. Uemura, N. et al. Inoculation of $\alpha$-synuclein preformed fibrils into the mouse gastrointestinal tract induces Lewy body-like aggregates in the brainstem via the vagus nerve. Molecular Neurodegeneration 13, 21 (2018).

67. Svensson, E. et al. Vagotomy and subsequent risk of Parkinson's disease. Ann Neurol. 78, 522$529(2015)$.

68. Tysnes, O.-B. et al. Does vagotomy reduce the risk of Parkinson's disease? Ann. Neurol. 78, 1011-1012 (2015).

69. Villumsen, M., Aznar, S., Pakkenberg, B., Jess, T. \& Brudek, T. Inflammatory bowel disease increases the risk of Parkinson's disease: a Danish nationwide cohort study 1977-2014. Gut 68, $18-24$ (2018).

70. Jung, S. et al. Analysis of fractalkine receptor CX(3)CR1 function by targeted deletion and green fluorescent protein reporter gene insertion. Mol. Cell. Biol. 20, 4106-4114 (2000).

71. Kitazawa, M., Oddo, S., Yamasaki, T. R., Green, K. N. \& LaFerla, F. M. Lipopolysaccharideinduced inflammation exacerbates tau pathology by a cyclin-dependent kinase 5-mediated pathway in a transgenic model of Alzheimer's disease. J. Neurosci. 25, 8843-8853 (2005).

72. Schenk, M., Bouchon, A., Seibold, F. \& Mueller, C. TREM-1--expressing intestinal macrophages crucially amplify chronic inflammation in experimental colitis and inflammatory bowel diseases. J. Clin. Invest. 117, 3097-3106 (2007).

73. Grathwohl, S. A. et al. Formation and maintenance of Alzheimer's disease $\beta$-amyloid plaques in the absence of microglia. Nat Neurosci 12, 1361-1363 (2009). 
813 74. Rey, N. L. et al. Spread of aggregates after olfactory bulb injection of $\alpha$-synuclein fibrils is

814 associated with early neuronal loss and is reduced long term. Acta Neuropathol 1-19 (2017).

815 doi:10.1007/s00401-017-1792-9

816 75. Schneider, C. A., Rasband, W. S. \& Eliceiri, K. W. NIH Image to ImageJ: 25 years of image

817 analysis. Nat. Methods 9, 671-675 (2012).

818 
Grathwohl et al., Figure 1
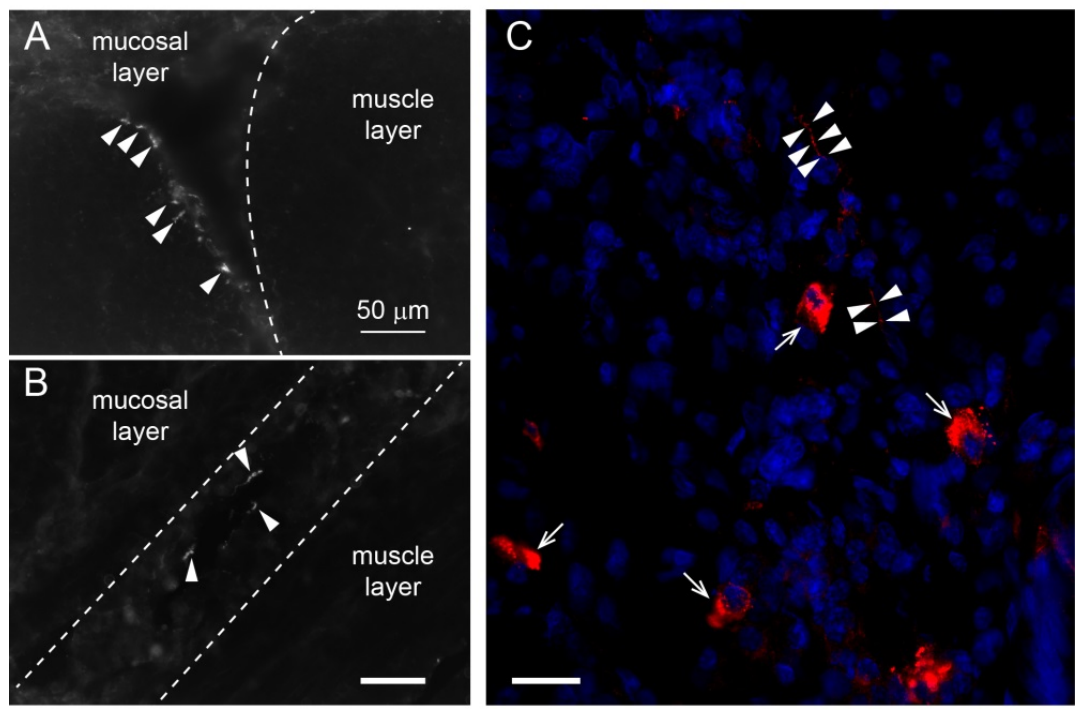

\section{$821 \quad$ Figure 1}

822 Alpha-Synuclein inclusions in the enteric nervous system and in macrophages of patients with

823 inflammatory bowel disease. (A, B) Immunofluorescence images of $\alpha$ Syn inclusions (Syn1

824 antibody) in the submucosal region of $10 \mu \mathrm{m}$ cryo-sections from colons of patients with colitis

825 ulcerosa. Arrow heads point to neuritic features indicating presence of inclusions in enteric nerves.

826 Scale bar $50 \mu \mathrm{m}$. (C) Close-up of a colonic region with active leukocyte infiltration in a patient with

827 ulcerative colitis. Immunoreactivity for $\alpha$ Syn (red) was observed in neuritic features (arrow heads) as

828 well as in individual leukocytes (arrows; identification of leukocytes based on cellular morphological

829 features and localization). Nuclei are shown in blue (DAPI). Scale bar $20 \mu \mathrm{m}$. 
bioRxiv preprint doi: https://doi.org/10.1101/505164: this version posted April 28,2019 . The copyright holder for this preprint (which was not certified by peer review) is the author/funder, who has granted bioRxiv a license to display the preprint in perpetuity. It is made available under aCC-BY-NC-ND 4.0 International license.

Grathwohl et al., Figure 2
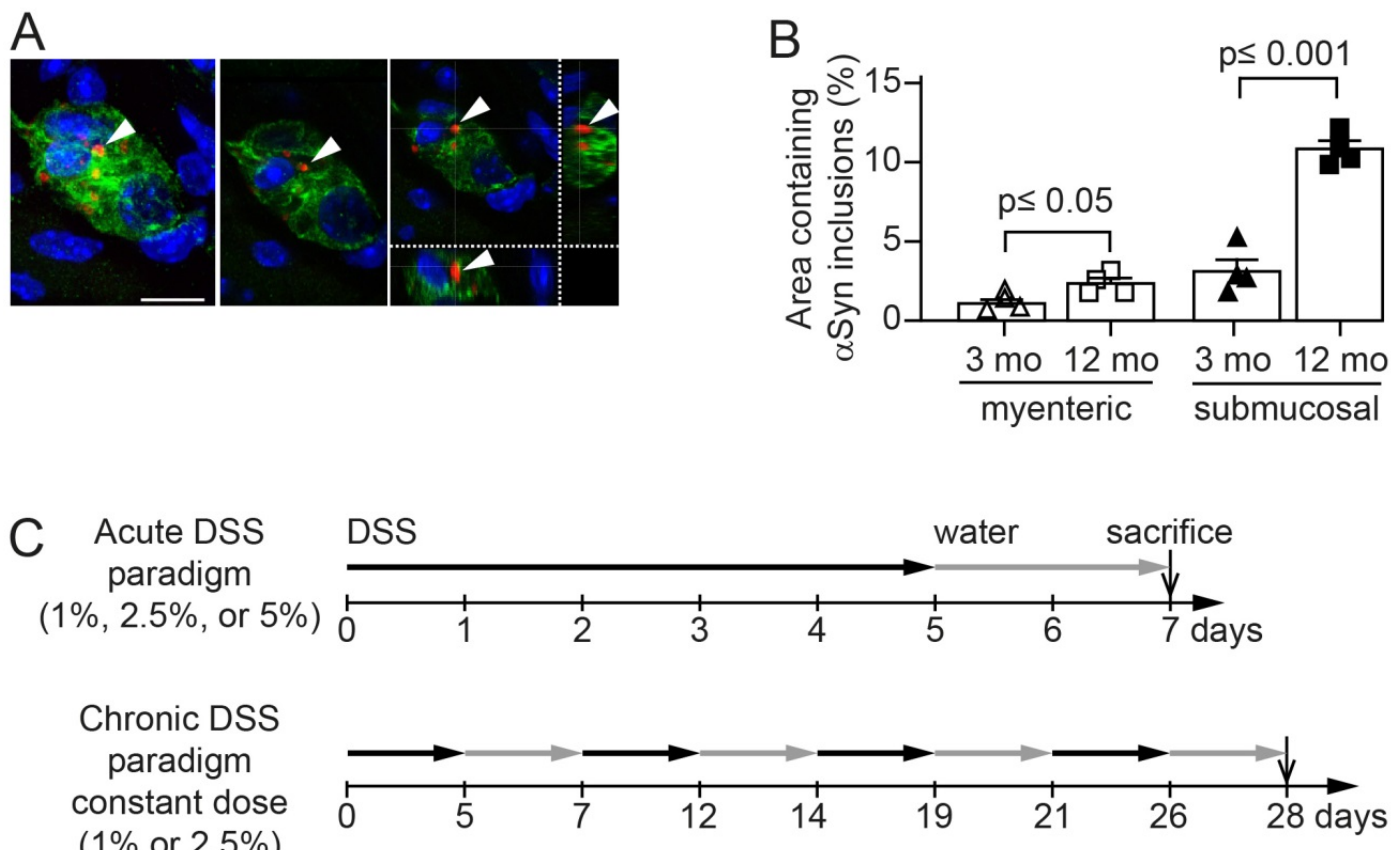

$(1 \%$ or $2.5 \%)$
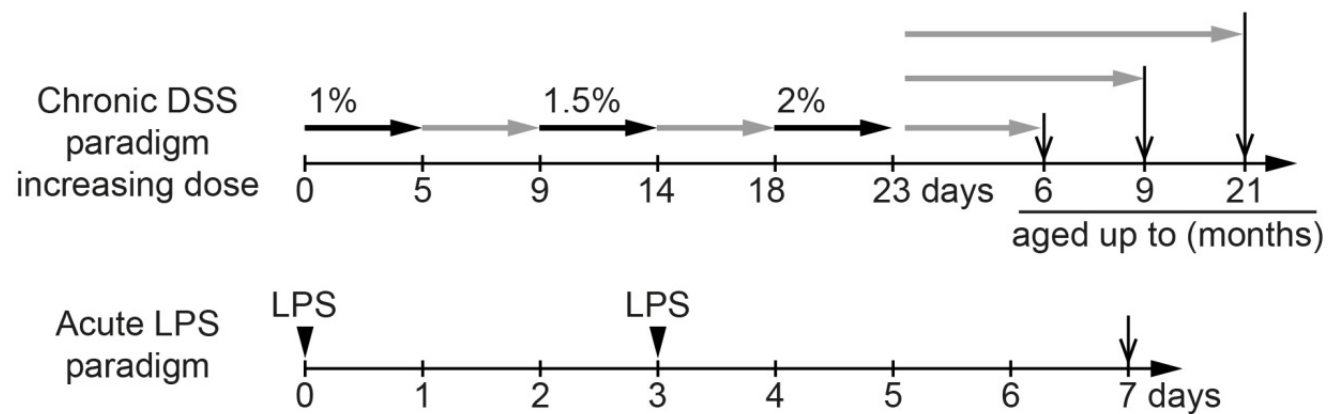

D

Intact colon

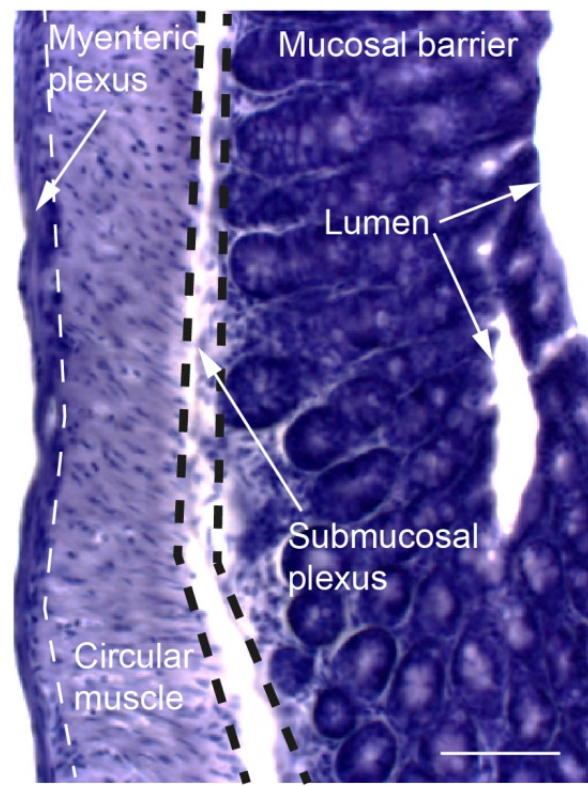

Acute DSS

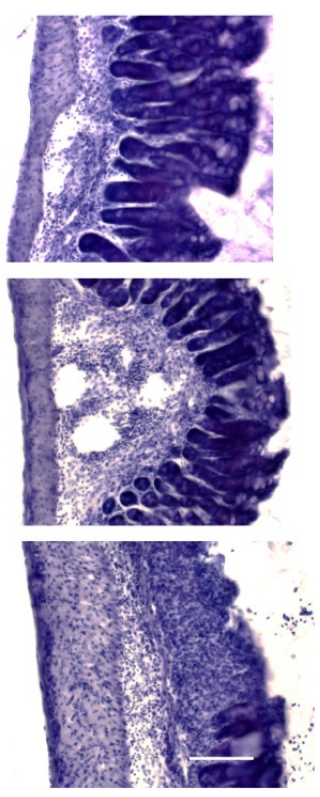

Acute LPS
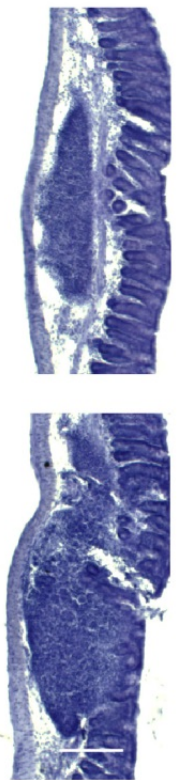


\section{Figure 2}

836 Age dependent increase of intracellular $\alpha$ Syn accumulation in enteric nervous system of 837 heterozygous (Thy1)-h[A30P] $\mathbf{S S y n}$ transgenic mice and setup of the experimental colitis paradigms. (A) Confocal microscopy imaging of the inclusions of human $\alpha$ Syn (red, antibody clone 211; human $\alpha$ Syn specific) within the ganglia of the submucosal plexus (green, peripherin; blue, DAPI/nuclei) of heterozygous (Thy1)-h[A30P] $\alpha$ Syn transgenic mice. Arrow head points to one of the typical irregularly sized and shaped $\alpha$ Syn inclusion bodies visualized in $2 \mathrm{D}$ z-stacks of rotated confocal images. Scale bar, $100 \mu \mathrm{m}$. (B) Stereological quantification of normally occurring human $\alpha$ Syn inclusions in the myenteric and submucosal plexuses of 3 and 12 months old heterozygous (Thy1)-h[A30P] $\alpha$ Syn transgenic mice ( $\mathrm{n}=4$ per group; mean and S.E.M. are shown; Student t-test between the two age groups in each region). (C) Setup of experimental colitis paradigms employing dextran sulfate sodium (DSS, per os in drinking water). Additionally, peripheral inflammation was induced by bacterial lipopolysaccharide (LPS, intraperitoneal injection). After some chronic DSS paradigms mice were aged on normal water up to 6,9 or 21 months. Mice aged up to 9 or 21 months of age were analyzed for brain pathology (D) Hematoxylin staining of $35 \mu \mathrm{m}$ thick colon sections of 3 months old heterozygous (Thy1)-h[A30P] $\alpha$ Syn transgenic mice. Organizational layers of the intact colon (left panel). Representative images of various severity degrees of DSS-driven colitis from weak leukocyte infiltration (top panel of acute DSS) to mucosal ulceration (lowest panel of acute DSS).

853 Note the different appearance of enteric inflammation in acute LPS-driven peripheral inflammation compared with DSS; e.g., confined immune cell clustering and lymphoid hyperplasia; intact mucosal layer. Scale bar $50 \mu \mathrm{m}$ (intact colon), $100 \mu \mathrm{m}$ (acute DSS), and $200 \mu \mathrm{m}$ (LPS). 
Grathwohl et al., Figure 3

A

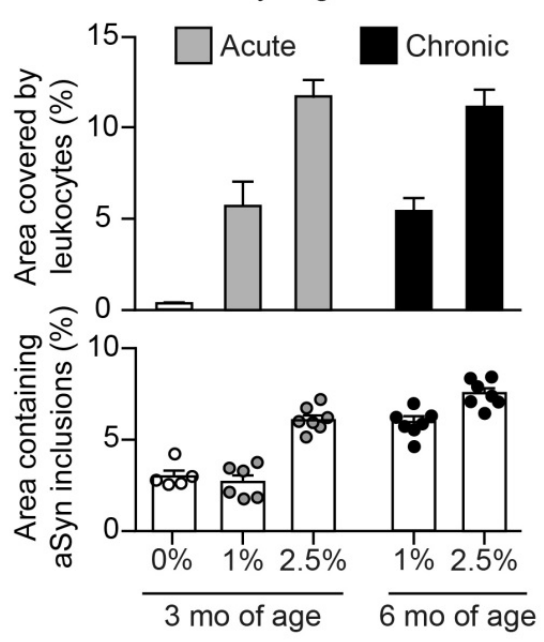

$\mathrm{B}$
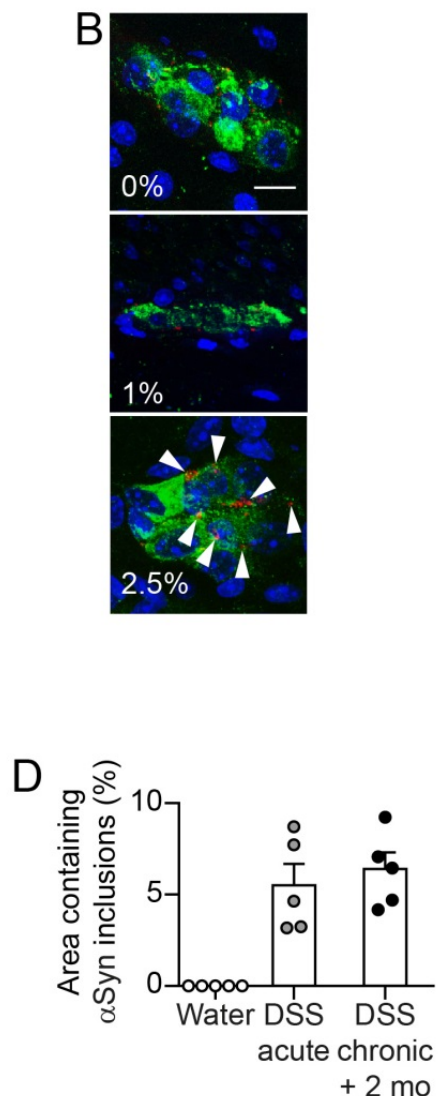

C

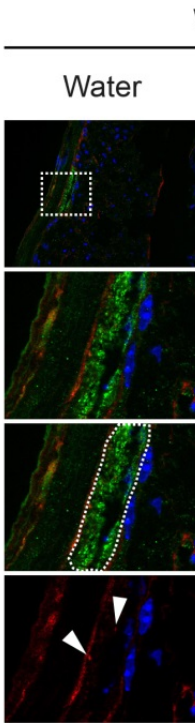

Wild type mice
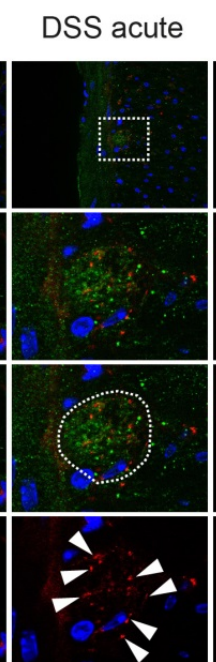

DSS chronic
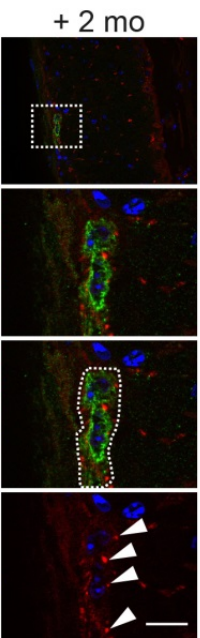

Figure 3

Colitis severity and duration-dependent aggravation of accumulation of $\alpha \mathrm{Syn}$ inclusions in the

(A) DSS dose-dependent increase of leukocyte infiltration in the acute and chronic paradigm. The highest acute dose (2.5\%) and the two constant chronic doses led to an increase of $\alpha$ Syn inclusions in the submucosal plexus (stereological quantification of $\alpha$ Syn inclusions in the submucosal plexus of 3 and 6 months old heterozygous (Thy1)-h[A30P $] \alpha$ Syn transgenic mice; $\mathrm{n}=5-7$ per group; mean and s.e.m. are shown). (B) Representative 2D z-stacks of confocal images of increasing abundance of 
$867 \alpha$ Syn inclusions (red, human- $\alpha$ Syn specific monoclonal antibody clone 211) in a ganglion of the

868 submucosal plexus (green, peripherin) with cellular nuclei in blue (DAPI) in the acute DSS paradigm.

869 Arrow heads point to the typical irregularly sized and shaped $\alpha$ Syn inclusion bodies that accumulate

870 in the highest DSS dose. Scale bar $200 \mu \mathrm{m}$. (C) Overview of colonic region of 3-month-old wildtype

871 mice (top row) exposed to water or acute DSS (5\%) with immunofluorescence analysis of murine

$872 \alpha$ Syn load in the colon performed immediately after colitis or exposed to constant chronic DSS (2.5\%)

873 and analysis after aging on normal water for another 2 months. White dotted rectangles in the top row

874 indicate the area that was zoomed out below illustrating in more detail the murine $\alpha$ Syn inclusions

875 (red, rodent $\alpha$ Syn cross-reactive monoclonal antibody syn1/clone 42) in the submucosal plexus

876 (green, peripherin). The lower three rows shows DAPI and $\alpha$ Syn inclusions with and without the

877 peripherin channel. The white dotted circled area illustrates the peripherin-positive area that was

878 analyzed for $\alpha$ Syn inclusion bodies (arrow heads in bottom row). Scale bar for the lower three panels

$879200 \mu \mathrm{m}$. (D) Stereological quantification of murine $\alpha$ Syn inclusions in the submucosal plexus of

880 wildtype mice right after acute DSS colitis or after 2 months of recovery from a 4-week chronic DSS

881 colitis ( $\mathrm{n}=5$ per group). Note the regularly arranged and smoothly distributed immunoreactivity for

882 the physiological $\alpha$ Syn with barely any inclusion bodies in the intact enteric nerves of the water

883 group.

884

885 
Grathwohl et al., Figure 4
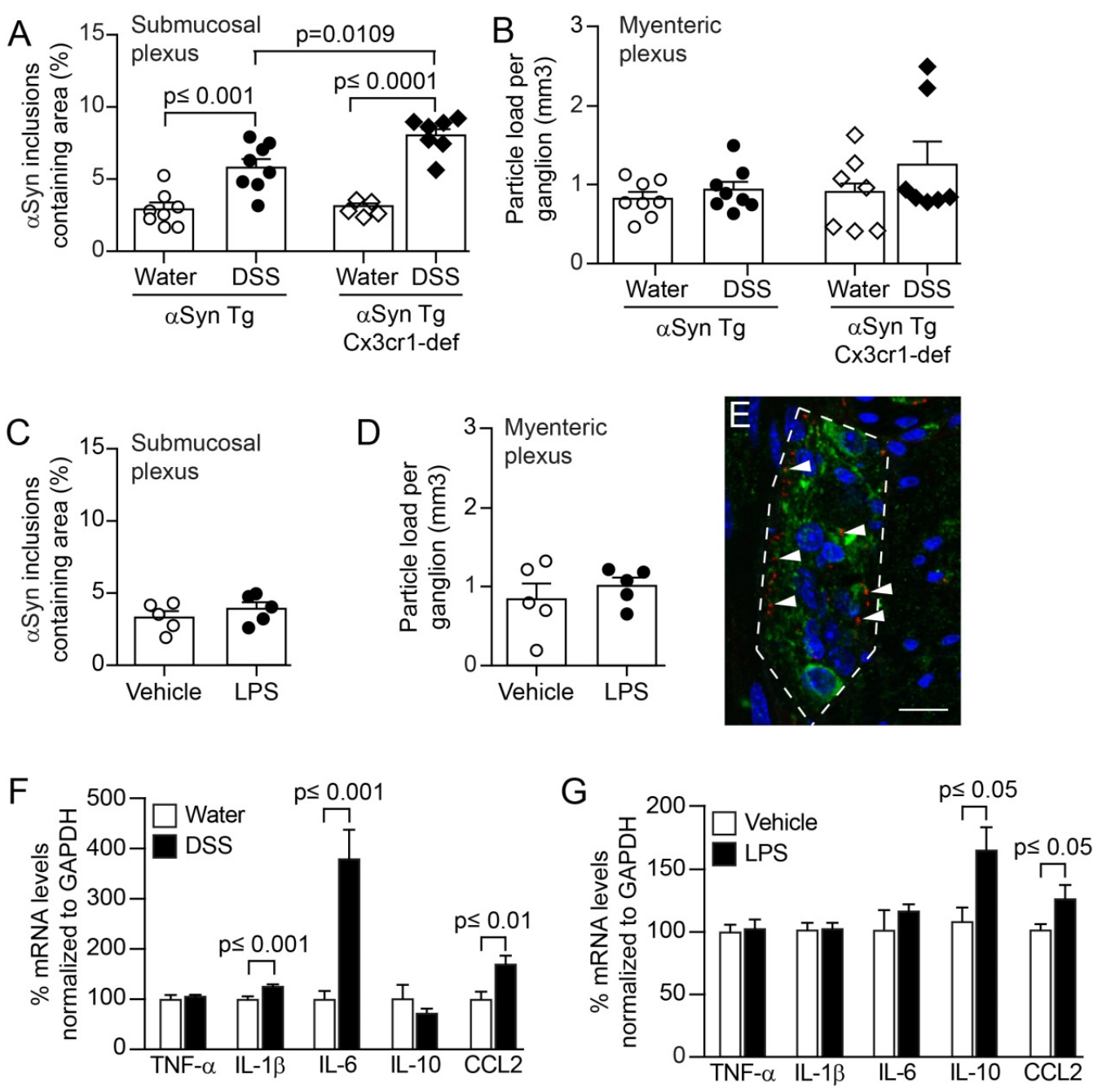

\section{$888 \quad$ Figure 4}

\section{Colitis induced by peroral DSS but not peritoneal LPS enhances $\alpha$ Syn accumulation in the} compared to effects induced by vehicle (see Figure 2C for timelines). Stereological quantification of $\alpha$ Syn inclusions in the submucosal plexus as \% area (A, C) and in the mucosal plexus as particle load per ganglion (B, D) (Two-way ANOVA with Tukey post hoc test). (E) Representative 2D stacks of confocal images of intracellular $\alpha$ Syn inclusions (red, human $\alpha$ Syn specific monoclonal antibody 
898 (green, peripherin) with cellular nuclei in blue (DAPI). Scale bar $50 \mu \mathrm{m}$. Gene expression analysis of

899 selected cytokines in the colon of (Thy1)-h[A30P] $\alpha$ Syn transgenic mice that received either acutely

900 LPS (F) or DSS (G) compared to their respective vehicle or water controls. Note the strong increase

901 in IL-6 and the lack of elevation of IL-10 in the DSS paradigm compared to the LPS paradigm

902 indicating a different inflammatory colonic milieu despite the abundant leukocyte infiltration in both

903 paradigms. $\mathrm{n}=5-8$ per group; mean and s.e.m.; Student's t-test between inflammatory agent and

904 vehicle for individual cytokines.

905

906

Grathwohl et al., Figure 5
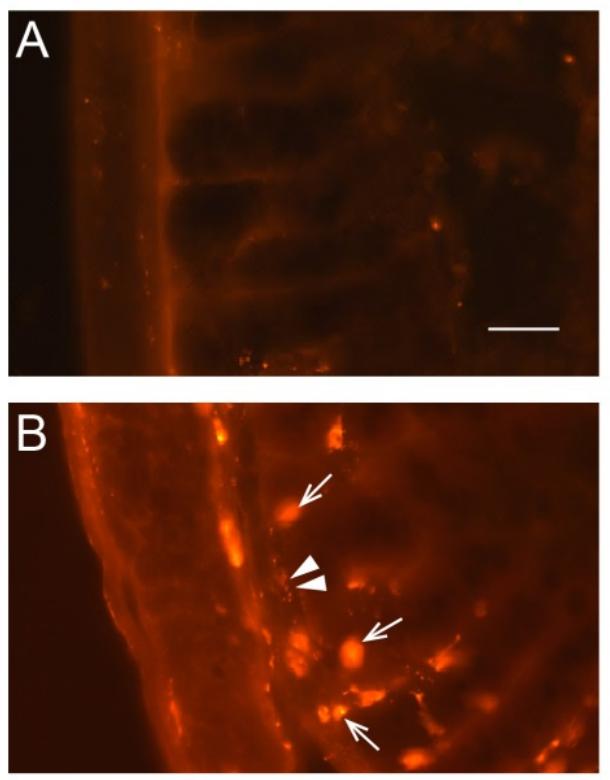
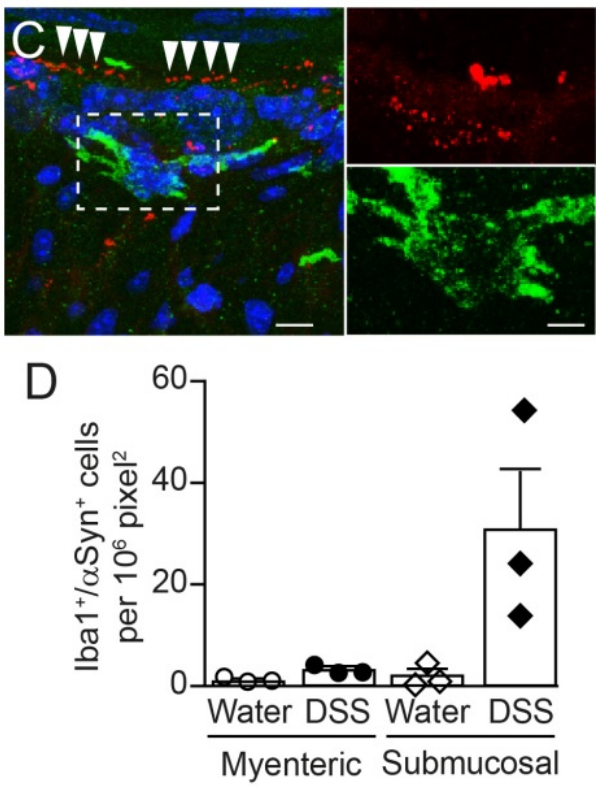

$908 \quad$ Figure 5

909 Alpha-synuclein co-localizes with ENS and macrophages upon DSS colitis in aSyn transgenic

910 mice. (A, B) Immunofluorescence image of $\alpha$ Syn staining in colonic region of (Thy1)-h[A30P] $\alpha$ Syn

911 transgenic mice on water (A) or after acute DSS colitis (2.5\%) (B). Note the small dotted structures of

912 the typical $\alpha$ Syn inclusions in the submucosal plexus (arrow heads) and the large features of

913 immunoreactivity which localize to infiltrating leukocytes (arrows; identified by their typical cellular

914 morphology), similar to what was observed in IBD patients in Figure 1. Scale bar $100 \mu \mathrm{m}$. (C) 2D 
915 stacks and close-up of confocal images co-localizing $\alpha$ Syn (red) with the macrophage marker Iba-1

916 (green) in the colon of a (Thy1)-h[A30P] Syn transgenic mouse after DSS colitis. Note the dotted

917 structures of the typical $\alpha$ Syn inclusions in the submucosal plexus (arrow heads). Scale bar $40 \mu \mathrm{m}$ and

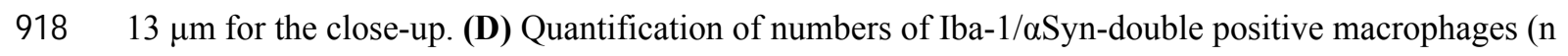

$919=3$ per group; mean and S.E.M.)

920

921

Grathwohl et al., Figure 6

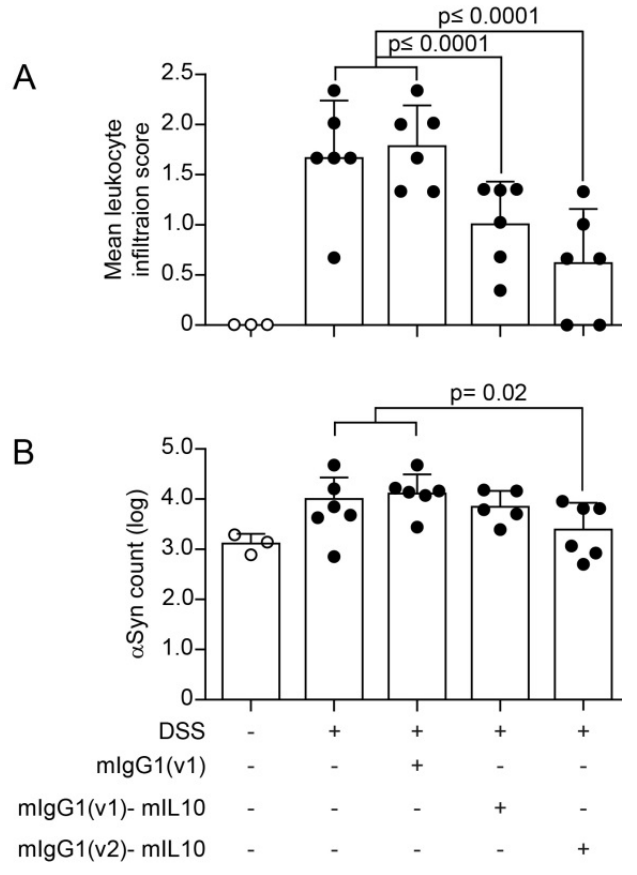

C

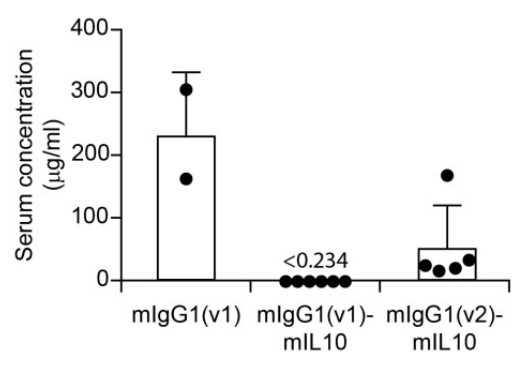

\section{$923 \quad$ Figure 6}

Systemic IL-10 ameliorates DSS colitis and associated local $\alpha$ Syn accumulation in (Thy1)-

$925 \mathbf{h}[\mathbf{A 3 0 P}] \boldsymbol{\alpha S y n}$ transgenic mice. Two different recombinantly engineered and murine IgG1-fused

926 forms of murine IL-10 (mIgG1(v1)-mIL10 and mIgG1(v2)-mIL10) were administered (150 $\mu \mathrm{g}$ per 
927 mouse i.p.) at the beginning of the acute DSS paradigm (5\%) in (Thy 1)-h[A30P] $\alpha$ Syn transgenic

928 mice. Vehicle and the $\mathrm{mIgG1}(\mathrm{v} 1)$ alone served as untreated controls. (A) Leukocyte infiltration was

929 assessed by visual scoring and (B) inclusion features of $\alpha$ Syn were stereologically and semi-

930 automatically quantified and result $\log$ scaled for statistical analysis. Both the vehicle group and the

931 mIgG1(v1) group had similar levels of leukocyte infiltration and $\alpha$ Syn inclusions and were merged for

932 the statistical analysis to compare with the IL-10 treated groups. Both forms of IL-10 ameliorated

933 leukocyte infiltration whereas mIgG1(v2)-mIL10 also blocked the appearance of $\alpha$ Syn inclusions

934 significantly ( $\mathrm{n}=3-6$ per group; mean and s.e.m.; one-way ANOVA and Tukey post hoc test). (C)

935 Persistent exposure mIgG1(v2)-mIL10 versus mIgG1(v1)-mIL10 (lower limit of detection is indicated

936 at $<0.234 \mu \mathrm{g} / \mathrm{ml}$ ) as measured in serum at the end of the in vivo phase corresponds with beneficial

937 treatment effects on $\alpha$ Syn readout observed above. The mIgG1(v1) was only measured in two mice. 
Grathwohl et al., Figure 7
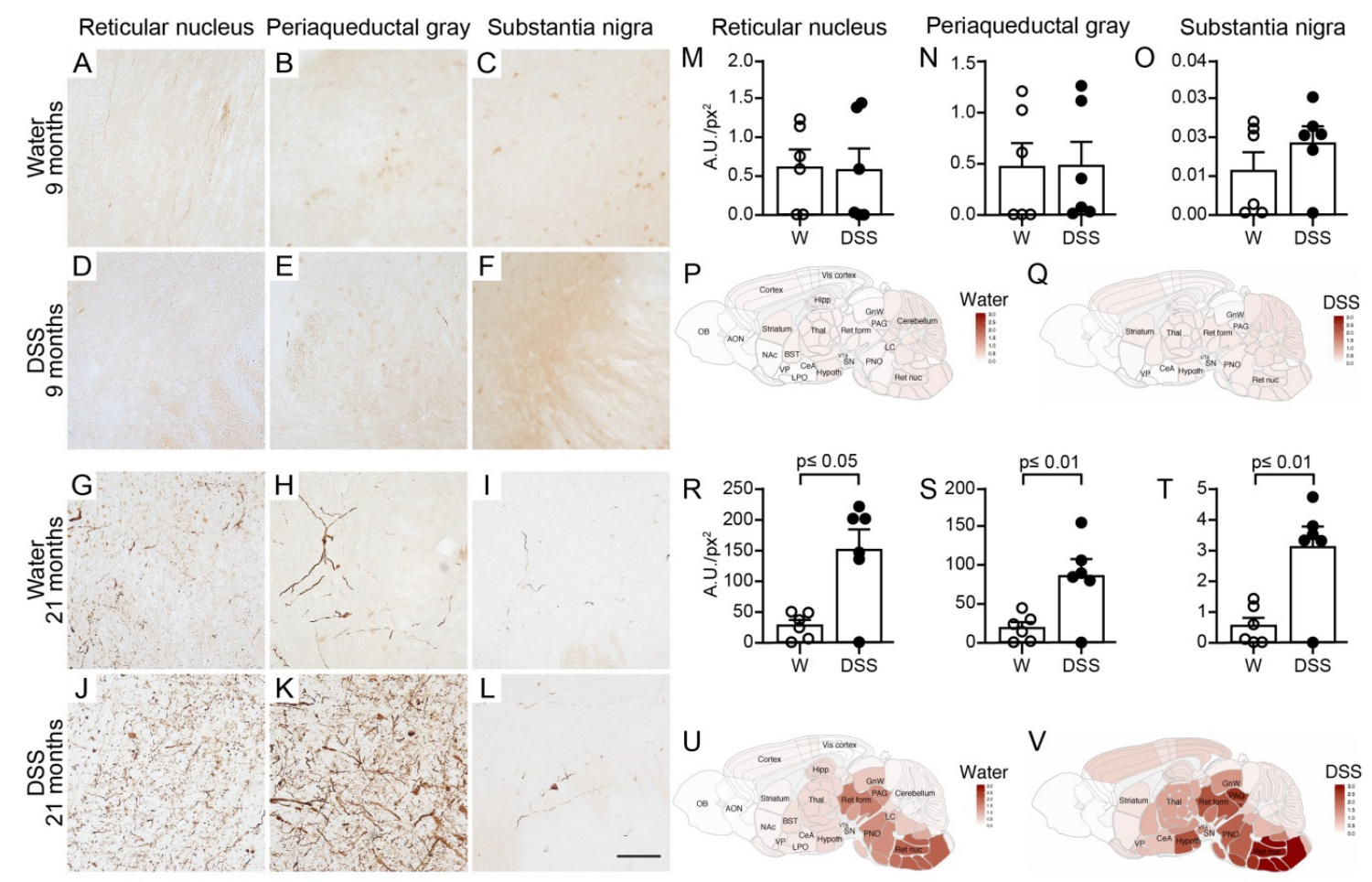

$943 \quad$ Figure 7

\section{A single chronic DSS colitis insult causes an age-dependent accumulation of proteinase $\mathrm{K}$} resistant pSer129-aSyn in various brain regions of (Thy1)-h[A30P] $\alpha$ Syn transgenic mice. A 3-

week chronic increasing dose DSS paradigm was performed with 3-month old (Thy1)-h[A30P] 2 Syn

transgenic mice. After recovering and further aging, various brain regions were analyzed for proteinase K resistant pSer129- $\alpha$ Syn immunoreactivity in 9-month (A-F) and 21-month old (G-L) mice, respectively. The dark brown features in G-L indicate proteinase K resistant pSer129- $\alpha$ Syn. They are barely visible in A-F. Densitometric quantification of pSer129- $\alpha$ Syn immunoreactivity in different brain regions in 9-month (M-O) and 21-month old mice (R-T) ( $\mathrm{n}=6$ mice per group). The two orders of magnitude different $\mathrm{y}$-axes between M-O and R-T confirm the visual impression in panel A-L. Statistical analyses were performed using negative-binomial mixed-effects models adjusting for multiple comparisons. Representative heatmap of the average distribution scores of pSer129- $\alpha$ Syn immunoreactivity for each treatment group in varying brain regions in all the 9-month 
956 (P-Q) and 21-month old (U-V) mice was generated in a sagittal mouse brain ( $\mathrm{n}=10$ mice per group).

957 Scale bars: $500 \mu \mathrm{m}$.

958

959

Grathwohl et al., Figure 8

A Substantia nigra level 1

Water

DSS

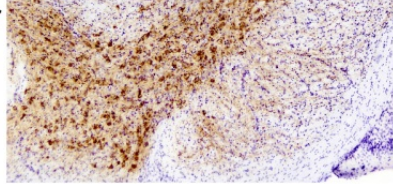

961

\section{Figure 8}

Substantia nigra level 2

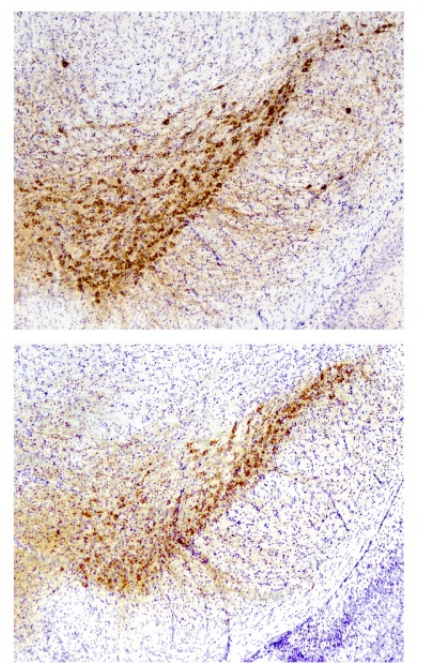

B

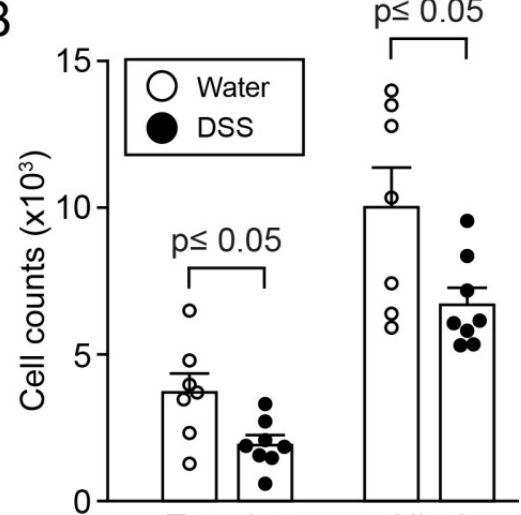

Tyrosine

Nissl hydroxylase

\section{Loss of tyrosine hydroxylase and Nissl positive cells in the substantia nigra of (Thy1)-}

\section{$\mathrm{h}[\mathrm{A30P}] \alpha$ Syn transgenic mice at 21 months of age, 18 months post recovery from DSS colitis.}

(Thy1)-h[A30P] $\alpha$ Syn transgenic mice that were exposed to a chronic DSS-colitis paradigm at 3

months and were aged to 21 months showed a significant loss of mean count of cells with tyrosine hydroxylase (TH) immunoreactivity and cellular Nissl staining in the substantia nigra compared to age-matched littermate mice in the group that did not experience DSS colitis (water). (A)

Representative images of two levels of the substantia nigra in one mouse per group. (B) Stereological quantification of cells positive for TH or Nissl ( $\mathrm{n}=7-8$ mice per group). Statistical analyses of the TH dataset were performed using Student's T-test, while Welch's T-test was used for the Nissl dataset to adjust for unequal variances. Scale bar: $500 \mu \mathrm{m}$. 